\title{
Cosine Similarity Measures of Bipolar Neutrosophic Set for Diagnosis of Bipolar Disorder Diseases
}

\author{
Mohamed Abdel-Basset *1, Mai Mohamed ', Mohamed Elhoseny 2, Le Hoang Son 3, Francisco \\ Chiclana ${ }^{4}$,Abd El-Nasser H Zaied ${ }^{1}$ \\ ${ }^{1}$ Faculty of Computers and Informatics, Zagazig University, Sharqiyah, Egypt; E-mail: \\ analyst_mohamed@zu.edu.eg; mmgafar@fci.zu.edu.eg;nasshzaied@zu.edu.eg \\ ${ }^{2}$ Faculty of Computers and Information, Mansoura University, Egypt; E-mail: mohamed_elhoseny@mans.edu.eg \\ ${ }^{3}$ VNU Information Technology Institute, Vietnam National University, Hanoi, Vietnam; E-mail: \\ sonlh@vnu.edu.vn \\ ${ }^{4}$ Anna University, India; E-mail: varathu21@yahoo.com \\ ${ }^{4}$ School of Computer Science and Informatics, De Montfort University, the Gateway, Leicester, LE1 9BH UK; \\ E-mail: chiclana@dmu.ac.uk
}

\begin{abstract}
Similarity plays a significant implicit or explicit role in various fields. In some real applications in decision making, similarity may bring counterintuitive outcomes from the decision maker's standpoint. Therefore, in this research, we propose some novel similarity measures for bipolar and interval-valued bipolar neutrosophic set such as the cosine similarity measures and weighted cosine similarity measures. The propositions of these similarity measures are examined, and two multi-attribute decision making techniques are presented based on proposed measures. For verifying the feasibility of proposed measures, two numerical examples are presented in comparison with the related methods for demonstrating the practicality of the proposed method. Finally, we applied the proposed measures of similarity for diagnosing bipolar disorder diseases.
\end{abstract}

Keywords: Bipolar; Cosine similarity measure; Multi-attribute decision making; Bipolar disorder diseases.

\section{Introduction}

The tendency of the human mind to realize and construct decision on the fundament of positive and negative impacts [31] is the main motivation for introducing the bipolar fuzzy set $(B F S)[32,33]$. In BFS, positive statements refer to what is probable, fitting, tolerable, or preferred. Meanwhile, negative statements imply what is impossible, discarded, or prohibited. Negative preferences compatible with constraints, because they identify which objects have to be discarded (i.e., those which do not meet constraints). On the other hand, positive preferences compatible with wishes, because they identify objects that are more eligible than others (i.e., suit wishes) without dismissing objects that do not convene the wishes.

Because of BFS's potentiality, many authors have studied bipolar fuzzy models on algebraic structures such as the concepts of bipolar fuzzy relations [31], $m$-polar fuzzy set [34], bipolar fuzzy groups, fuzzy d-ideals of groups under (T-S) norm [35], bipolar valued fuzzy sub-semigroup, bipolar valued fuzzy bi-ideal, and bipolar valued fuzzy ideal [36], bipolar fuzzy sets in sub-semirings [37], and others [38-40, 52-57]. For handling imprecision and uncertainty which exist in real life, neutrosophic set (NS) concept, which is a popularization of fuzzy and intuitionistic fuzzy set theory [1, 2], was presented in [3]. 
Since NS has the ability to consider incomplete, imprecise and conflicting information [3], the concept of bipolar neutrosophic set (BNS) has been presented [41]. It is a crossbreeding of bipolar fuzzy set and NS since each element has three independent positive and negative membership functions.

There is practically little literature on the applications of BNS on algebraic structures. A decision making approach based on Jaccard vector similarity has been studied in [42]. A multi-attribute decision making (MADM) approach based on dice similarity measure has been studied in [43]. Also, the concept of interval valued bipolar neutrosophic set was presented in [44]. Several authors have used these models of sets in diverse application fields [4-30, 50-57].

In bipolar neutrosophic sets, cosine similarity measure is yet to appear in literature. Thus in this research, we present new cosine similarity measures for the bipolar neutrosophic set (BNS) and interval valued bipolar neutrosophic set (IVBNS). We present three novel similarity measures based on the cosine function and their weighted similarity measures for bipolar and interval valued bipolar neutrosophic sets. We also developed two MADM methods under bipolar and interval valued bipolar neutrosophic sets. We also solved two practical problems and compared results with other existing measures of similarity. Finally, we applied proposed measures for diagnosing bipolar disorder disease, which is a brain disorder that creates uncommon moves in energy, mood, and the ability to perform daily tasks.

The structure of this research is as follows: Section 2 introduces some basic concepts and definitions of BNSs and IVBNSs. Section 3 presents the drawbacks of some existing similarity measures for BNSs. Section 4 devotes similarity measures based on cosine function for bipolar neutrosophic sets and interval valued bipolar neutrosophic sets. Section 5 presents two MADM techniques based on proposed measures. Section 6 demonstrates two numerical examples and compares results with other presented methods. Section 7 presents a medical diagnosis problem of bipolar disorder disease based on proposed measures of similarity. Section 8 provides concluding observations and future trends of this research.

\section{Concepts and definitions of bipolar neutrosophic set}

We recollect some definitions that are associated with the present research in this section.

\subsection{Bipolar neutrosophic set [41]}

A bipolar neutrosophic set (BNS) $B I$ in $X$ is defined as an object of the form:

$$
B I=\left\langle x\left(\Gamma^{+}{ }_{B I}(x), \xi^{+}{ }_{B I}(x), \Psi^{+}{ }_{B I}(x), \Gamma^{-}{ }_{B I}(x), \xi^{-}{ }_{B I}(x), \Psi^{-}{ }_{B I}(x)\right) \mid x \in X\right\rangle .
$$

Here, $\Gamma^{+}{ }_{B I}(x), \xi^{+}{ }_{B I}(x), \Psi^{+}{ }_{B I}(x): B I \rightarrow[0,1]$ and $\Gamma_{B I}^{-}(x), \xi^{-}{ }_{B I}(x), \Psi^{-}{ }_{B I}(x): B I \rightarrow$ $[-1,0], \Gamma^{+}, \xi^{+}, \Psi^{+}$stand for truth, indeterminate and falsity membership degrees of an element $x \in X$, and similarly the negative membership degrees $\Gamma^{-}, \xi^{-}, \Psi^{-}$. 


\subsection{Basic properties of bipolar neutrosophic set [41]}

Assume that $B I_{1}=$ $\left\{\left\langle x\left(\Gamma_{B I_{1}}^{+}(x), \xi^{+}{ }_{B I_{1}}(x), \Psi^{+}{ }_{B I_{1}}(x), \Gamma^{-}{ }_{B I_{1}}(x), \xi^{-}{ }_{B I_{1}}(x), \Psi^{-}{ }_{B I_{1}}(x)\right)\right\rangle \mid x \in X\right\}$, and $B I_{2}=$ $\left\{\left\langle x\left(\Gamma^{+}{ }_{B I_{2}}(x), \xi^{+}{ }_{B I_{2}}(x), \Psi^{+}{ }_{B I_{2}}(x), \Gamma^{-}{ }_{B I_{2}}(x), \xi^{-}{ }_{B I_{2}}(x), \Psi^{-}{ }_{B I_{2}}(x)\right)\right\rangle \mid x \in X\right\}$ be two bipolar neutrosophic sets then,

1) $B I_{1}=B I_{2}$ if and only if, $\Gamma^{+}{ }_{B I_{1}}(x)=\Gamma^{+}{ }_{B I_{2}}(x), \xi^{+}{ }_{B I_{1}}(x)=$ $\xi^{+}{ }_{B I_{2}}(x), \Psi^{+}{ }_{B I_{1}}(x)=\Psi^{+}{ }_{B I_{2}}(x)$ $, \Gamma^{-}{ }_{B I_{1}}(x)=\Gamma^{-}{ }_{B I_{2}}(x), \xi^{-}{ }_{B I_{1}}(x)=\xi^{-}{ }_{B I_{2}}(x), \Psi^{-}{ }_{B I_{1}}(x)=\Psi^{-}{ }_{B I_{2}}(x)$.

2) $B I_{1} \cup B I_{2}=$ $\operatorname{Max}\left(\Gamma^{+}{ }_{B I_{1}}(x), \Gamma^{+}{ }_{B I_{2}}(x)\right), \frac{\xi_{B I_{1}}^{+}(x)+\xi^{+}{ }_{B I_{2}}(x)}{2}, \operatorname{Min}\left({\Psi^{+}}_{B I_{1}}(x), \Psi^{+}{ }_{B I_{2}}(x)\right)$, $\operatorname{Min}\left(\Gamma^{-}{ }_{B I_{1}}(x), \Gamma^{-}{ }_{B I_{2}}(x)\right), \frac{\xi_{B I_{1}}^{-}(x)+\xi^{-}{ }_{B I_{2}}(x)}{2}, \operatorname{Max}\left({\Psi^{-}}_{B I_{1}}(x), \Psi^{-}{ }_{B I_{2}}(x)\right)$.

3) $B I_{1} \cap B I_{2}=$ $\operatorname{Min}\left(\Gamma^{+}{ }_{B I_{1}}(x), \Gamma^{+}{ }_{B I_{2}}(x)\right), \frac{\xi_{B I_{1}}^{+}(x)+\xi^{+}{ }_{B I_{2}}(x)}{2}, \operatorname{Max}\left({\Psi^{+}}_{B I_{1}}(x),{\Psi^{+}}_{B I_{2}}(x)\right)$, $\operatorname{Max}\left(\Gamma^{-}{ }_{B I_{1}}(x), \Gamma^{-}{ }_{B I_{2}}(x)\right), \frac{\xi^{-}{ }_{B I_{1}}(x)+\xi^{-}{ }_{B I_{2}}(x)}{2}, \operatorname{Min}\left({\Psi^{-}}_{B I_{1}}(x), \Psi^{-}{ }_{B I_{2}}(x)\right)$.

4) $B I^{C}=\left\langle\begin{array}{c}1-\Gamma^{+}{ }_{B I}(x), 1-\xi^{+}{ }_{B I}(x), 1-\Psi^{+}{ }_{B I}(x), 1-\Gamma^{-}{ }_{B I}(x), 1-\xi^{-}{ }_{B I}(x) \\ 1-\Psi^{-}{ }_{B I}(x)\end{array}\right\rangle$.

5) $B I_{1} \subseteq B I_{2}$ if and only if, $\Gamma^{+}{ }_{B I_{1}}(x) \leq \Gamma^{+}{ }_{B I_{2}}(x), \xi^{+}{ }_{B I_{1}}(x) \leq$ $\xi^{+}{ }_{B I_{2}}(x), \Psi^{+}{ }_{B I_{1}}(x) \geq \Psi^{+}{ }_{B I_{2}}(x), \Gamma^{-}{ }_{B I_{1}}(x) \geq \Gamma^{-}{ }_{B I_{2}}(x), \xi^{-}{ }_{B I_{1}}(x) \geq$ $\xi^{-}{ }_{B I_{2}}(x), \Psi^{-}{ }_{B I_{1}}(x) \leq \Psi^{-}{ }_{B I_{2}}(x)$.

\subsection{Interval valued bipolar neutrosophic set [44]}

An interval valued bipolar neutrosophic set (IVBNS) $I V$ in $X$ has the following form:

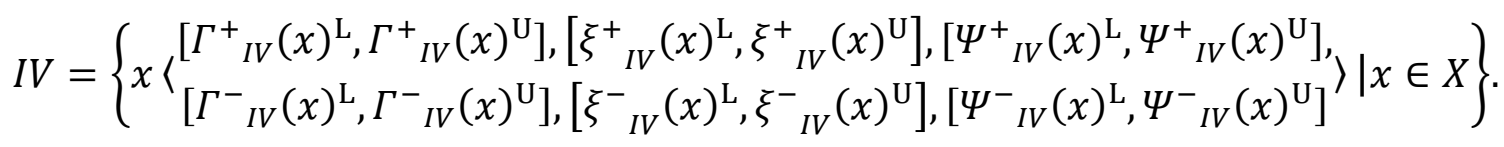

Since, $\Gamma^{+}{ }_{I V}(x)^{\mathrm{L}}, \Gamma^{+}{ }_{I V}(x)^{\mathrm{U}}, \xi^{+}{ }_{I V}(x)^{\mathrm{L}}, \xi^{+}{ }_{I V}(x)^{\mathrm{U}}, \Psi^{+}{ }_{I V}(x)^{\mathrm{L}}, \Psi^{+}{ }_{I V}(x)^{\mathrm{U}}: I V \rightarrow[0,1] \quad$ and $\Gamma_{I V}^{-}(x)^{\mathrm{L}}, \Gamma^{-}{ }_{I V}(x)^{\mathrm{U}}, \xi^{-}{ }_{I V}(x)^{\mathrm{L}}, \xi^{-}{ }_{I V}(x)^{\mathrm{U}}, \Psi^{-}{ }_{I V}(x)^{\mathrm{L}}, \Psi^{-}{ }_{I V}(x)^{\mathrm{U}}: I V \rightarrow[-1,0]$.

In IVBNS, the positive and negative membership degrees are in interval form instead of a fixed value of an element $x \in X$. 


\subsection{Basic properties of interval valued bipolar neutrosophic set [44]}

Assume that, $I V_{1}=$

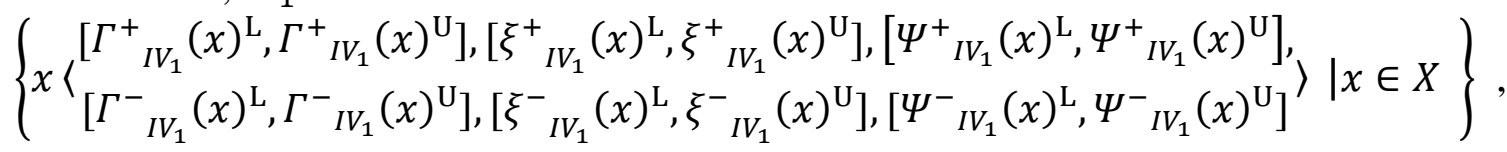

and

$I V_{2}=$

$\left\{\begin{array}{c}{\left[\Gamma_{{ }^{+}{ }_{I V_{2}}}(x)^{\mathrm{L}}, \Gamma^{+}{ }_{I V_{2}}(x)^{\mathrm{U}}\right],\left[\xi^{+}{ }_{I V_{2}}(x)^{\mathrm{L}}, \xi^{+}{ }_{I V_{2}}(x)^{\mathrm{U}}\right],\left[\Psi^{+}{ }_{I V_{2}}(x)^{\mathrm{L}}, \Psi^{+}{ }_{I V_{2}}(x)^{\mathrm{U}}\right],} \\ {\left[\Gamma^{-}{ }_{I V_{2}}(x)^{\mathrm{L}}, \Gamma^{-}{ }_{I V_{2}}(x)^{\mathrm{U}}\right],\left[\xi^{-}{ }_{I V_{2}}(x)^{\mathrm{L}}, \xi^{-}{ }_{I V_{2}}(x)^{\mathrm{U}}\right],\left[\Psi^{-}{ }_{I V_{2}}(x)^{\mathrm{L}}, \Psi^{-}{ }_{I V_{2}}(x)^{\mathrm{U}}\right]}\end{array}\right\}$

be two interval valued bipolar neutrosophic sets. Then,

1) $I V_{1}=I V_{2}$ if and only if, $\Gamma^{+}{ }_{I V_{1}}(x)^{\mathrm{L}}=\Gamma^{+}{ }_{I V_{2}}(x)^{\mathrm{L}}, \Gamma^{+}{ }_{I V_{1}}(x)^{\mathrm{U}}=$

$\Gamma_{{ }^{+} V_{2}}(x)^{\mathrm{U}}, \xi^{+}{ }_{I V_{1}}(x)^{\mathrm{L}}=\xi^{+}{ }_{I V_{2}}(x)^{\mathrm{L}}$,

$\xi^{+}{ }_{I V_{1}}(x)^{\mathrm{U}}=\xi^{+}{ }_{I V_{2}}(x)^{\mathrm{U}}, \Psi^{+}{ }_{I V_{1}}(x)^{\mathrm{L}}=\Psi^{+}{ }_{I V_{2}}(x)^{\mathrm{L}}, \Psi^{+}{ }_{I V_{1}}(x)^{\mathrm{U}}=\Psi^{+}{ }_{I V_{2}}(x)^{\mathrm{U}}$,

$\Gamma^{-}{ }_{I V_{1}}(x)^{\mathrm{L}}=\Gamma^{-}{ }_{I V_{2}}(x)^{\mathrm{L}}, \Gamma^{-}{ }_{I V_{1}}(x)^{\mathrm{U}}=\Gamma^{-}{ }_{I V_{2}}(x)^{\mathrm{U}}, \xi^{-}{ }_{I V_{1}}(x)^{\mathrm{L}}=\xi^{-}{ }_{I V_{2}}(x)^{\mathrm{L}}$,

$\xi^{-}{ }_{I V_{1}}(x)^{\mathrm{U}}=\xi^{-}{ }_{I V_{2}}(x)^{\mathrm{U}}, \Psi^{-{ }_{I V_{1}}}(x)^{\mathrm{L}}=\Psi^{-}{ }_{I V_{2}}(x)^{\mathrm{L}}, \Psi^{-{ }_{I V_{1}}}(x)^{\mathrm{U}}=\Psi^{-}{ }_{I V_{2}}(x)^{\mathrm{U}}$.

2) $I V_{1} \cup I V_{2}=$

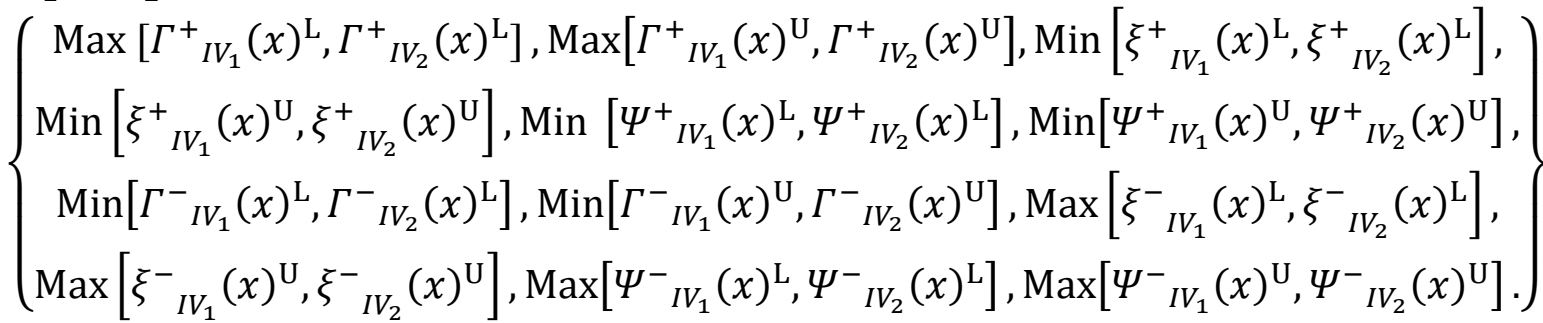

3) $I V_{1} \cap I V_{2}=$

$\left\{\begin{array}{c}\operatorname{Min}\left[\Gamma^{+}{ }_{I V_{1}}(x)^{\mathrm{L}}, \Gamma^{+}{ }_{I V_{2}}(x)^{\mathrm{L}}\right], \operatorname{Min}\left[\Gamma^{+}{ }_{I V_{1}}(x)^{\mathrm{U}}, \Gamma^{+}{ }_{I V_{2}}(x)^{\mathrm{U}}\right], \operatorname{Max}\left[\xi^{+}{ }_{I V_{1}}(x)^{\mathrm{L}}, \xi^{+}{ }_{I V_{2}}(x)^{\mathrm{L}}\right], \\ \operatorname{Max}\left[\xi^{+}{ }_{I V_{1}}(x)^{\mathrm{U}}, \xi^{+}{ }_{I V_{2}}(x)^{\mathrm{U}}\right], \operatorname{Max}\left[{\Psi^{+}}^{+}{ }_{I V_{1}}(x)^{\mathrm{L}},{\Psi^{+}}{ }_{I V_{2}}(x)^{\mathrm{L}}\right], \operatorname{Max}\left[\Psi^{+}{ }_{I V_{1}}(x)^{\mathrm{U}}, \Psi^{+}{ }_{I V_{2}}(x)^{\mathrm{U}}\right], \\ \operatorname{Max}\left[\Gamma^{-}{ }_{I V_{1}}(x)^{\mathrm{L}}, \Gamma^{-}{ }_{I V_{2}}(x)^{\mathrm{L}}\right], \operatorname{Max}\left[\Gamma^{-}{ }_{I V_{1}}(x)^{\mathrm{U}}, \Gamma^{-}{ }_{I V_{2}}(x)^{\mathrm{U}}\right], \operatorname{Min}\left[\xi^{-}{ }_{I V_{1}}(x)^{\mathrm{L}}, \xi^{-}{ }_{I V_{2}}(x)^{\mathrm{L}}\right], \\ \operatorname{Min}\left[\xi^{-}{ }_{I V_{1}}(x)^{\mathrm{U}}, \xi^{-}{ }_{I V_{2}}(x)^{\mathrm{U}}\right], \operatorname{Min}\left[{\Psi^{-}}_{{ }_{I V}}(x)^{\mathrm{L}}, \Psi^{-}{ }_{I V_{2}}(x)^{\mathrm{L}}\right], \min \left[\Psi^{-}{ }_{I V_{1}}(x)^{\mathrm{U}}, \Psi^{-}{ }_{I V_{2}}(x)^{\mathrm{U}}\right] .\end{array}\right\}$

4) $I V^{C}=$

$\left\{\begin{array}{c}{\left[1-\Gamma^{+}{ }_{I V}(x)^{\mathrm{L}}, 1-\Gamma^{+}{ }_{I V}(x)^{\mathrm{U}}\right],\left[1-\xi^{+}{ }_{I V}(x)^{\mathrm{L}}, 1-\xi^{+}{ }_{I V}(x)^{\mathrm{U}}\right],} \\ {\left[1-\Psi^{+}{ }_{I V}(x)^{\mathrm{L}}, 1-\Psi^{+}{ }_{I V}(x)^{\mathrm{U}}\right],\left[-1-\Gamma^{-}{ }_{I V}(x)^{\mathrm{L}},-1-\Gamma^{-}{ }_{I V}(x)^{\mathrm{U}}\right],} \\ {\left[-1-\xi^{-}{ }_{I V}(x)^{\mathrm{L}},-1-\xi^{-}{ }_{I V}(x)^{\mathrm{U}}\right],\left[-1-\Psi^{-}{ }_{I V}(x)^{\mathrm{L}},-1-\Psi^{-}{ }_{I V}(x)^{\mathrm{U}}\right]}\end{array}\right\}$

5) $I V_{1} \subseteq I V_{2}$ if and only if, $\Gamma^{+}{ }_{I V_{1}}(x)^{\mathrm{L}} \leq \Gamma^{+}{ }_{I V_{2}}(x)^{\mathrm{L}}, \Gamma^{+}{ }_{I V_{1}}(x)^{\mathrm{U}} \leq$

$\Gamma^{+}{ }_{I V_{2}}(x)^{\mathrm{U}}, \xi^{+}{ }_{I V_{1}}(x)^{\mathrm{L}} \geq \xi^{+}{ }_{I V_{2}}(x)^{\mathrm{L}}$,

$\xi^{+}{ }_{I V_{1}}(x)^{\mathrm{U}} \geq \xi^{+}{ }_{I V_{2}}(x)^{\mathrm{U}}, \Psi^{++}{ }_{I V_{1}}(x)^{\mathrm{L}} \geq \Psi^{+}{ }_{{ } V_{2}}(x)^{\mathrm{L}}, \Psi^{+}{ }_{I V_{1}}(x)^{\mathrm{U}} \geq \Psi^{+}{ }_{I V_{2}}(x)^{\mathrm{U}}$,

$\Gamma^{-}{ }_{I V_{1}}(x)^{\mathrm{L}} \geq \Gamma^{-}{ }_{I V_{2}}(x)^{\mathrm{L}}, \Gamma^{-}{ }_{I V_{1}}(x)^{\mathrm{U}} \geq \Gamma^{-}{ }_{I V_{2}}(x)^{\mathrm{U}}, \xi^{-}{ }_{I V_{1}}(x)^{\mathrm{L}} \leq \xi^{-}{ }_{I V_{2}}(x)^{\mathrm{L}}$

, $\xi^{-}{ }_{I V_{1}}(x)^{\mathrm{U}} \leq \xi^{-}{ }_{I V_{2}}(x)^{\mathrm{U}}, \Psi^{-}{ }_{I V_{1}}(x)^{\mathrm{L}} \leq \Psi^{-}{ }_{I V_{2}}(x)^{\mathrm{L}}, \Psi^{-}{ }_{I V_{1}}(x)^{\mathrm{U}} \leq \Psi^{-}{ }_{I V_{2}}(x)^{\mathrm{U}}$. 


\section{Drawbacks of the existing measures of similarity for BNSs}

In this part, we present some measures of similarity for bipolar neutrosophic set in the literature and illustrate their drawbacks.

Firstly, the dice similarity measure among two bipolar neutrosophic sets $G, H$ which presented by Ulucay et al. [43] and denoted as $D(G, H)$;

$$
\begin{aligned}
& D(G, H)=\frac{1}{n} \sum_{i=1}^{n} \times \\
& \left(\begin{array}{c}
\frac{\left[\left(\Gamma^{+}{ }_{G}\left(x_{i}\right) \Gamma^{+}{ }_{H}\left(x_{i}\right)+\xi^{+}{ }_{G}\left(x_{i}\right) \xi^{+}{ }_{H}\left(x_{i}\right)+\Psi^{+}{ }_{G}\left(x_{i}\right) \Psi^{+}{ }_{H}\left(x_{i}\right)\right)-\left(\Gamma^{-}{ }_{G}\left(x_{i}\right) \Gamma^{-}{ }_{H}\left(x_{i}\right)+\xi^{-}{ }_{G}\left(x_{i}\right) \xi^{-}{ }_{H}\left(x_{i}\right)+\Psi^{-}{ }_{G}\left(x_{i}\right) \Psi^{-}{ }_{H}\left(x_{i}\right)\right)\right]}{\left[\left(\left(\Gamma^{+}{ }_{G}\right)^{2}\left(x_{i}\right)+\left(\xi^{+}{ }_{G}\right)^{2}\left(x_{i}\right)+\left(\Psi^{+}{ }_{G}\right)^{2}\left(x_{i}\right)\right)+\left(\left(\Gamma^{+}{ }_{H}\right)^{2}\left(x_{i}\right)+\left(\xi^{+}{ }_{H}\right)^{2}\left(x_{i}\right)+\left(\Psi^{+}{ }_{H}\right)^{2}\left(x_{i}\right)\right)\right.} \\
\left.-\left(\left(\Gamma^{-}{ }_{G}\right)^{2}\left(x_{i}\right)+\left(\xi^{-}{ }_{G}\right)^{2}\left(x_{i}\right)+\left(\Psi^{-}{ }_{G}\right)^{2}\left(x_{i}\right)\right)-\left(\left(\Gamma^{-}{ }_{H}\right)^{2}\left(x_{i}\right)+\left(\xi^{-}{ }_{H}\right)^{2}\left(x_{i}\right)+\left(\Psi^{-}{ }_{H}\right)^{2}\left(x_{i}\right)\right)\right]
\end{array}\right)
\end{aligned}
$$

The properties of previous similarity measure are as follows:

1) $0 \leq D(G, H) \leq 1$;

2) $D(G, H)=D(H, G)$

3) $D(G, H)=1$ for $G=H$.

The deficiencies in Eq. (1) are as follows:

1) For two bipolar neutrosophic sets $G$ and $H$, if $G=\{\langle(1,1,0,-1,-1,0)\rangle\}$ and $H=$ $\{\langle(0,1,1,-1,-1,0)\rangle\}$ then,

$D(G, H)=\frac{1}{6} \times$

$\left(\frac{[(0+1+0)-(1+1+0)]}{[(1+1+0)+(0+1+1)-(1+1+0)-(1+1+0)]}\right)=\frac{1}{6} \times\left(\frac{-1}{0}\right)$, which is undefined or unmeaningful and in this case, it cannot be used for calculating similarity measure among $G$ and $H$.

2) If $G=\langle(1,1,0,-1,-1,0)\rangle, H=\langle(1,1,0,-1,-1,0)\rangle$ and it is obvious that two equal bipolar neutrosophic set, then in this case the $D(G, H)$ must equal one according to third property, and by applying Eq. (1) then,

$D(G, H)=\frac{1}{6} \times\left(\frac{[(1+1+0)-(1+1+0)]}{[(1+1+0)+(1+1+0)-(1+1+0)-(1+1+0)]}\right)=\frac{1}{6} \times\left(\frac{0}{0}\right)=0$, which contrasts with third property of previous similarity measure, since the result must equal 1.

The same drawbacks appear also in weighted dice similarity measure [43] among $G$ and $H$ denoted by $D_{w}(G, H)$; 


$$
\begin{aligned}
& D_{w}(G, H)= \\
& \sum_{i=1}^{n} w_{i} \times\left(\begin{array}{c}
\frac{\left[\left(\Gamma^{+}{ }_{G}\left(x_{i}\right) \Gamma^{+}{ }_{H}\left(x_{i}\right)+\xi^{+}{ }_{G}\left(x_{i}\right) \xi^{+}{ }_{H}\left(x_{i}\right)+\Psi^{+}{ }_{G}\left(x_{i}\right) \Psi^{+}{ }_{H}\left(x_{i}\right)\right)-\left(\Gamma^{-}{ }_{G}\left(x_{i}\right) \Gamma^{-}{ }_{H}\left(x_{i}\right)+\xi^{-}{ }_{G}\left(x_{i}\right) \xi^{-}{ }_{H}\left(x_{i}\right)+\Psi^{-}{ }_{G}\left(x_{i}\right) \Psi^{-}{ }_{H}\left(x_{i}\right)\right)\right]}{\left[\left(\left(\Gamma^{+}{ }_{G}\right)^{2}\left(x_{i}\right)+\left(\xi^{+}{ }_{G}\right)^{2}\left(x_{i}\right)+\left(\Psi^{+}{ }_{G}\right)^{2}\left(x_{i}\right)\right)+\left(\left(\Gamma^{+}{ }_{H}\right)^{2}\left(x_{i}\right)+\left(\xi^{+}{ }_{H}\right)^{2}\left(x_{i}\right)+\left(\Psi^{+}{ }_{H}\right)^{2}\left(x_{i}\right)\right)\right.} \\
\left.-\left(\left(\Gamma^{-}{ }_{G}\right)^{2}\left(x_{i}\right)+\left(\xi^{-}{ }_{G}\right)^{2}\left(x_{i}\right)+\left({ }^{-}{ }_{G}\right)^{2}\left(x_{i}\right)\right)-\left(\left(\Gamma^{-}{ }_{H}\right)^{2}\left(x_{i}\right)+\left(\xi^{-}{ }_{H}\right)^{2}\left(x_{i}\right)+\left(\Psi^{-}{ }_{H}\right)^{2}\left(x_{i}\right)\right)\right]
\end{array}\right)
\end{aligned}
$$

The properties of previous similarity measure are as follows:
1) $0 \leq D_{w}(G, H) \leq 1$
2) $D_{w}(G, H)=D_{w}(H, G)$;
3) $D_{w}(G, H)=1$ for $G=H$.

The deficiencies in Eq. (2) are as follows:

1) For two bipolar neutrosophic sets $G$ and $H$, if $G=\{\langle(1,1,0,-1,-1,0)\rangle\}$ and $H=$ $\{\langle(0,1,1,-1,-1,0)\rangle\}$, let $w=0.3$ then, $D_{w}(G, H)=0.3 \times\left(\frac{[(0+1+0)-(1+1+0)]}{[(1+1+0)+(0+1+1)-(1+1+0)-(1+1+0)]}\right)=0.3 \times\left(\frac{-1}{0}\right)$, which is undefined or un-meaningful and in this case Eq.(2) cannot used for calculating weighted dice similarity measure among $G$ and $H$.

2) If $G=\langle(1,1,0,-1,-1,0)\rangle, H=\langle(1,1,0,-1,-1,0)\rangle$ and two equals bipolar neutrosophic set, and $w=0.3$, then in this case the $D_{w}(G, H)$ must equal one and by applying Eq. (2) then,

$D_{w}(G, H)=0.3 \times\left(\frac{[(1+1+0)-(1+1+0)]}{[(1+1+0)+(1+1+0)-(1+1+0)-(1+1+0)]}\right)=0.3 \times\left(\frac{0}{0}\right)=0$, and this contrasts with third property of previous similarity measure, since the result must equal 1.

The previous drawbacks appear also in the hybrid vector similarity measure [43] which is as follows: $\operatorname{HybV}(G, H)=\lambda \frac{1}{\mathrm{n}} \times$

$$
\begin{aligned}
& \sum_{i=1}^{n}\left(\begin{array}{c}
\frac{\left[\left(\Gamma^{+}{ }_{G}\left(x_{i}\right) \Gamma^{+}{ }_{H}\left(x_{i}\right)+\xi^{+}{ }_{G}\left(x_{i}\right) \xi^{+}{ }_{H}\left(x_{i}\right)+\Psi^{+}{ }_{G}\left(x_{i}\right) \Psi^{+}{ }_{H}\left(x_{i}\right)\right)-\left(\Gamma^{-}{ }_{G}\left(x_{i}\right) \Gamma^{-}{ }_{H}\left(x_{i}\right)+\xi^{-}{ }_{G}\left(x_{i}\right) \xi^{-}{ }_{H}\left(x_{i}\right)+\psi^{-}{ }_{G}\left(x_{i}\right) \Psi^{-}{ }_{H}\left(x_{i}\right)\right)\right]}{2\left[\left(\left(\Gamma^{+}{ }_{G}\right)^{2}\left(x_{i}\right)+\left(\xi^{+}{ }_{G}\right)^{2}\left(x_{i}\right)+\left(\Psi^{+}{ }_{G}\right)^{2}\left(x_{i}\right)\right)+\left(\left(\Gamma^{+}{ }_{H}\right)^{2}\left(x_{i}\right)+\left(\xi^{+}{ }_{H}\right)^{2}\left(x_{i}\right)+\left(\Psi^{+}{ }_{H}\right)^{2}\left(x_{i}\right)\right)\right.} \\
\left.-\left(\left(\Gamma^{-}{ }_{G}\right)^{2}\left(x_{i}\right)+\left(\xi^{-}{ }_{G}\right)^{2}\left(x_{i}\right)+\left(\Psi^{-}{ }_{G}\right)^{2}\left(x_{i}\right)\right)-\left(\left(\Gamma^{-}{ }_{H}\right)^{2}\left(x_{i}\right)+\left(\xi^{-}{ }_{H}\right)^{2}\left(x_{i}\right)+\left(\Psi^{-}{ }_{H}\right)^{2}\left(x_{i}\right)\right)\right]
\end{array}\right) \\
& +(1-\lambda) \frac{1}{\mathrm{n}} \sum_{i=1}^{n} \times
\end{aligned}
$$

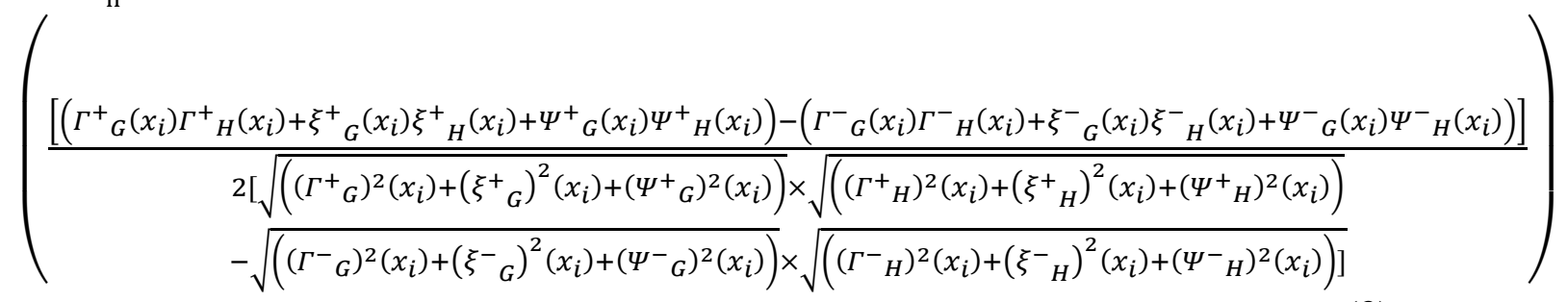


Beside the previous drawbacks in the weighted hybrid vector similarity measure, the different values of $\lambda$ will also make various results of ranking if it used for ranking process of alternatives. Then, we can say that the third problem of using Eq. (3) lies also in the acquisition of the exact value of parameter $\lambda$.

Also, there are some deficiencies in the proposed measures of similarity for BNSs presented by Sahin et. Al. [42] as follows: The Jaccard vector similarity measure among two bipolar neutrosophic set $G, H$ denoted as $J(G, H) . \quad J(G, H)=\frac{1}{n} \sum_{i=1}^{n} \times$ $\frac{1}{2}\left[\begin{array}{c}\frac{\left(\Gamma^{+}{ }_{G}\left(x_{i}\right) \Gamma^{+}{ }_{H}\left(x_{i}\right)+\xi^{+}{ }_{G}\left(x_{i}\right) \xi^{+}{ }_{H}\left(x_{i}\right)+\Psi^{+}{ }_{G}\left(x_{i}\right) \Psi^{+}{ }_{H}\left(x_{i}\right)\right)-\left(\Gamma^{-}{ }_{G}\left(x_{i}\right) \Gamma^{-}{ }_{H}\left(x_{i}\right)+\xi^{-}{ }_{G}\left(x_{i}\right) \xi^{-}{ }_{H}\left(x_{i}\right)+\Psi^{-}{ }_{G}\left(x_{i}\right) \Psi^{-}{ }_{H}\left(x_{i}\right)\right)}{\left(\Gamma^{+}{ }_{G}\right)^{2}\left(x_{i}\right)+\left(\xi^{+}{ }_{G}\right)^{2}\left(x_{i}\right)+\left(\Psi^{+}{ }_{G}\right)^{2}\left(x_{i}\right)+\left(\Gamma^{+}{ }_{H}\right)^{2}\left(x_{i}\right)+\left(\xi^{+}{ }_{H}\right)^{2}\left(x_{i}\right)+\left(\Psi^{+}{ }_{H}\right)^{2}\left(x_{i}\right)+\left(\Gamma^{-}{ }_{G}\right)^{2}\left(x_{i}\right)+\left(\xi^{-}{ }_{G}\right)^{2}\left(x_{i}\right)+\left(\Psi^{-}{ }_{G}\right)^{2}\left(x_{i}\right)+} \\ \left(\Gamma^{-}{ }_{H}\right)^{2}\left(x_{i}\right)+\left(\xi^{-}{ }_{H}\right)^{2}\left(x_{i}\right)+\left(\Psi^{-}{ }_{H}\right)^{2}\left(x_{i}\right)-\left(\Gamma^{+}{ }_{G}\left(x_{i}\right) \Gamma^{+}{ }_{H}\left(x_{i}\right)+\xi^{+}{ }_{G}\left(x_{i}\right) \xi^{+}{ }_{H}\left(x_{i}\right)+\Psi^{+}{ }_{G}\left(x_{i}\right) \Psi^{+}{ }_{H}\left(x_{i}\right)\right)- \\ \left(\Gamma^{-}{ }_{G}\left(x_{i}\right) \Gamma^{-}{ }_{H}\left(x_{i}\right)+\xi^{-}{ }_{G}\left(x_{i}\right) \xi^{-}{ }_{H}\left(x_{i}\right)+\Psi^{-}{ }_{G}\left(x_{i}\right) \Psi^{-}{ }_{H}\left(x_{i}\right)\right)\end{array}\right]$

The properties of previous similarity measure are as follows:

1) $0 \leq J(G, H) \leq 1$;

2) $J(G, H)=J(H, G)$;

3) $J(G, H)=1$ for $G=H$.

The deficiencies in Eq. (4) are as follows:

Let $G=\langle(1,0,0,-1,0,0)\rangle, H=\langle(1,0,0,-1,0,0)\rangle$, then in this case the $J(G, H)$ must equal 1 according to third property of this similarity measure. But by applying Eq. (4) we noted that $J(G, H)=0$, which conflicts with third property, since the result must equal 1 . The same drawback appears also in proposed weighted Jaccard similarity measure between $G$ and $H$.

Another drawback of Jaccard similarity measure is as follows:

Let $G=\langle(0,0,0,-1,0,0)\rangle$, and $H=\langle(0,0,0,-1,0,0)\rangle$. By applying Eq. (4) then, $J(G, H)=\frac{1}{2} \times-1=-\frac{1}{2}$, which also conflicts with first property of this similarity measure. The same drawback appears also in weighted Jaccard similarity measure between $G$ and $H$.

Cosine similarity measure is yet to appear in literature for bipolar and interval valued bipolar neutrosophic set, but appeared in literature for single valued neutrosophic set. For handling shortcoming of presented cosine similarity measures for single valued neutrosophic set, Ye [45] proposed two types of improved cosine similarity measures. But, his proposed measures of similarity (i.e. two types of improved cosine similarity measures) failed also to produce reasonable results:

The proposed types of improved cosine similarity measures between two single valued neutrosophic set $A$ and $B$ are as follows: 
$S c_{1}(A, B)=\frac{1}{n} \times \sum_{j=1}^{n} \cos \left[\frac{\pi\left(\left|\Gamma_{\mathrm{A}}\left(\mathrm{x}_{\mathrm{j}}\right)-\Gamma_{\mathrm{B}}\left(\mathrm{x}_{\mathrm{j}}\right)\right| \mathrm{v}\left|\xi_{\mathrm{A}}\left(\mathrm{x}_{\mathrm{j}}\right)-\xi_{\mathrm{B}}\left(\mathrm{x}_{\mathrm{j}}\right)\right| \mathrm{v}\left|\Psi_{\mathrm{A}}\left(\mathrm{x}_{\mathrm{j}}\right)-\Psi_{\mathrm{B}}\left(\mathrm{x}_{\mathrm{j}}\right)\right|\right.}{2}\right]$

$S c_{2}(A, B)=\frac{1}{n} \times \sum_{j=1}^{n} \cos \left[\frac{\pi\left(\left|\Gamma_{\mathrm{A}}\left(\mathrm{x}_{\mathrm{j}}\right)-\Gamma_{\mathrm{B}}\left(\mathrm{x}_{\mathrm{j}}\right)\right|+\left|\xi_{\mathrm{A}}\left(\mathrm{x}_{\mathrm{j}}\right)-\xi_{\mathrm{B}}\left(\mathrm{x}_{\mathrm{j}}\right)\right|+\left|\Psi_{\mathrm{A}}\left(\mathrm{x}_{\mathrm{j}}\right)-\Psi_{\mathrm{B}}\left(\mathrm{x}_{\mathrm{j}}\right)\right|\right.}{6}\right]$

The drawbacks of two types of cosine similarity measures are as follows:

Let $A=\langle 1,0,0\rangle$ and $=\langle 0,0,0\rangle$, by applying Eq. (5) and Eq. (6) then, $S c_{1}(A, B)=0$, although the two sets are similar in $\xi, \Psi$. But at the same situation the $S c_{2}(A, B)=$ 0.8660. Then, the low and high similarity value of $S c_{1}(A, B)$ and $S c_{2}(A, B)$, produces an unreasonable phenomenon for the similarity measures between $A$ and $B$. So, they are not appropriate to handle medical diagnosis problems.

For overcoming all drawbacks of previous similarity measures we proposed three types of similarity measures for BNSs and IVBNSs.

\section{Proposed measures of similarity for BNSs and IVBNSs}

In this part, we present new types of cosine similarity measures along with their weighted similarity measures of bipolar neutrosophic environments. We also compare proposed measures of similarity with other presented measures in literature.

\subsection{Similarity measures for BNSs and IVBNSs}

Definition 1. Let $B I_{1}=$

$\left\{\left\langle x\left(\Gamma_{B I_{1}}^{+}(x), \xi^{+}{ }_{B I_{1}}(x), \Psi^{+}{ }_{B I_{1}}(x), \Gamma^{-}{ }_{B I_{1}}(x), \xi^{-}{ }_{B I_{1}}(x), \Psi^{-}{ }_{B I_{1}}(x)\right)\right\rangle \mid x \in X\right\}, B I_{2}=$ $\left\{\left\langle x\left(\Gamma^{+}{ }_{B I_{2}}(x), \xi^{+}{ }_{B I_{2}}(x), \Psi^{+}{ }_{B I_{2}}(x), \Gamma^{-}{ }_{B I_{2}}(x), \xi^{-}{ }_{B I_{2}}(x), \Psi^{-}{ }_{B I_{2}}(x)\right)\right\rangle \mid x \in X\right\}$

be two bipolar neutrosophic sets in a universe of discourse $X$. Then, cosine similarity measure among bipolar neutrosophic sets $B I_{1}$ and $B I_{2}$ denoted by $C s_{t}\left(B I_{1}, B I_{2}\right), t=$ $1,2,3$ is defined as follows:

$$
\begin{aligned}
& C S_{1}\left(B I_{1}, B I_{2}\right)=\cos \left(\frac{\pi}{12} \times\left(\left|\Gamma^{+}{ }_{B I_{1}}(x)-\Gamma^{+}{ }_{B I_{2}}(x)\right|+\left|\xi^{+}{ }_{B I_{1}}(x)-\xi^{+}{ }_{B I_{2}}(x)\right|+\right.\right. \\
& \left|\Psi^{+}{ }_{B I_{1}}(x)-\Psi^{+}{ }_{B I_{2}}(x)\right|+\left|\Gamma^{-}{ }_{B I_{1}}(x)-\Gamma^{-}{ }_{B I_{2}}(x)\right|+\left|\xi^{-}{ }_{B I_{1}}(x)-\xi^{-}{ }_{B I_{2}}(x)\right|+ \\
& \left.\left.\left|\Psi^{-}{ }_{B I_{1}}(x)-\Psi^{-}{ }_{B I_{2}}(x)\right|\right)\right) \\
& C S_{2}\left(B I_{1}, B I_{2}\right)=\cos \left(\frac{\pi}{4} \times \operatorname{Max}\left(\left|\Gamma^{+}{ }_{B I_{1}}(x)-\Gamma^{+}{ }_{B I_{2}}(x)\right|, \mid \xi^{+}{ }_{B I_{1}}(x)-\right.\right. \\
& \xi^{+}{ }_{B I_{2}}(x)|,| \Psi^{+}{ }_{B I_{1}}(x)-\Psi^{+}{ }_{B I_{2}}(x)|,| \Gamma^{-}{ }_{B I_{1}}(x)-\Gamma^{-}{ }_{B I_{2}}(x)|,| \xi^{-}{ }_{B I_{1}}(x)- \\
& \left.\left.\xi_{B I_{2}}^{-}(x)|,| \Psi^{-}{ }_{B I_{1}}(x)-\Psi^{-}{ }_{B I_{2}}(x) \mid\right)\right)(8)
\end{aligned}
$$


$C s_{3}\left(B I_{1}, B I_{2}\right)=\cos \left(\frac{\pi}{2} \times\left(\frac{M_{1}+M_{2}}{2}\right)\right)$

Since,

$$
\begin{aligned}
& M_{1}= \\
& \operatorname{Max}\left(\begin{array}{l}
\left|\Gamma^{+}{ }_{B I_{1}}(x)-\Gamma^{+}{ }_{B I_{2}}(x)\right|,\left|\xi^{+}{ }_{B I_{1}}(x)-\xi^{+}{ }_{B I_{2}}(x)\right|,\left|\Psi^{+}{ }_{B I_{1}}(x)-\Psi^{+}{ }_{B I_{2}}(x)\right|, \\
\left|\Gamma^{-}{ }_{B I_{1}}(x)-\Gamma^{-}{ }_{B I_{2}}(x)\right|,\left|\xi^{-}{ }_{B I_{1}}(x)-\xi^{-}{ }_{B I_{2}}(x)\right|,\left|\Psi^{-}{ }_{B I_{1}}(x)-\Psi^{-}{ }_{B I_{2}}(x)\right|
\end{array}\right), \\
& M_{2}= \\
& \operatorname{Min}\left(\begin{array}{l}
\left|\Gamma^{+}{ }_{B I_{1}}(x)-\Gamma^{+}{ }_{B I_{2}}(x)\right|,\left|\xi^{+}{ }_{B I_{1}}(x)-\xi^{+}{ }_{B I_{2}}(x)\right|,\left|\Psi^{+}{ }_{B I_{1}}(x)-\Psi^{+}{ }_{B I_{2}}(x)\right|, \\
\left|\Gamma^{-}{ }_{B I_{1}}(x)-\Gamma^{-}{ }_{B I_{2}}(x)\right|,\left|\xi^{-}{ }_{B I_{1}}(x)-\xi^{-}{ }_{B I_{2}}(x)\right|,\left|\Psi^{-}{ }_{B I_{1}}(x)-\Psi^{-}{ }_{B I_{2}}(x)\right|
\end{array}\right) .
\end{aligned}
$$

The three types of similarity measures $C s_{t}\left(B I_{1}, B I_{2}\right), t=1,2,3$ meet these characteristics:

a) $0 \leq C s_{t}\left(B I_{1}, B I_{2}\right) \leq 1$,

Proof

For any bipolar neutrosophic set, $\Gamma^{+}(x), \xi^{+}(x)$, and $\Psi^{+}(x)$ lie within $[0,1]$ and $\Gamma^{-}(x), \xi^{-}(x)$, and $\Psi^{-}(x)$ lie within $[-1,0]$. So, the values of the suggested cosine functions are within $[0,1]$.

b) $C s_{t}\left(B I_{1}, B I_{2}\right)=C s_{t}\left(B I_{2}, B I_{1}\right), t=1,2,3$.

Proof

c) Since $\left|\Gamma^{+}{ }_{B I_{1}}(x)-\Gamma^{+}{ }_{B I_{2}}(x)\right|=\left|\Gamma^{+}{ }_{B I_{2}}(x)-\Gamma^{+}{ }_{B I_{1}}(x)\right|,\left|\xi^{+}{ }_{B I_{1}}(x)-\xi^{+}{ }_{B I_{2}}(x)\right|=$ $\left|\xi^{+}{ }_{B I_{2}}(x)-\xi^{+}{ }_{B I_{1}}(x)\right|,\left|\Psi^{+}{ }_{B I_{1}}(x)-\Psi^{+}{ }_{B I_{2}}(x)\right|=\left|\Psi^{+}{ }_{B I_{2}}(x)-\Psi^{+}{ }_{B I_{1}}(x)\right|=$ $\left|\Gamma^{-}{ }_{B I_{1}}(x)-\Gamma^{-}{ }_{B I_{2}}(x)\right|=\left|\Gamma^{-}{ }_{B I_{2}}(x)-\Gamma^{-}{ }_{B I_{1}}(x)\right|=\left|\xi^{-}{ }_{B I_{1}}(x)-\xi^{-}{ }_{B I_{2}}(x)\right|=$ $\left|\xi^{-}{ }_{B I_{2}}(x)-\xi^{-}{ }_{B I_{1}}(x)\right|=$ and $\left|\Psi^{-}{ }_{B I_{1}}(x)-\Psi^{-}{ }_{B I_{2}}(x)\right|=\left|\Psi^{-}{ }_{B I_{2}}(x)-\Psi^{-}{ }_{B I_{1}}(x)\right|$

Thus, $C s_{t}\left(B I_{1}, B I_{2}\right)=C s_{t}\left(B I_{2}, B I_{1}\right), t=1,2,3$.

d) $C s_{t}\left(B I_{1}, B I_{2}\right)=1$, if and only if $B I_{1}=B I_{2}$.

Proof

For any two bipolar neutrosophic set $B I_{1}$ and $B I_{2}$, when $B I_{1}=B I_{2}$ then,

$$
\begin{aligned}
& \Gamma^{+}{ }_{B 1}(x)=\Gamma^{+}{ }_{B I_{2}}(x), \xi^{+}{ }_{B I_{1}}(x)=\xi^{+}{ }_{B I_{2}}(x), \Psi^{+}{ }_{B I_{1}}(x)=\Psi^{+}{ }_{B I_{2}}(x), \Gamma^{-}{ }_{B I_{1}}(x) \\
& =\Gamma^{-}{ }_{B I_{2}}(x), \xi^{-}{ }_{B I_{1}}(x)=\xi^{-}{ }_{B I_{2}}(x), \text { and } \Psi^{-}{ }_{B I_{1}}(x)=\Psi^{-}{ }_{B I_{2}}(x) .
\end{aligned}
$$

Also,

$$
\left|\Gamma^{+}{ }_{B I_{1}}(x)-\Gamma^{+}{ }_{B I_{2}}(x)\right|=0,\left|\xi^{+}{ }_{B I_{1}}(x)-\xi^{+}{ }_{B I_{2}}(x)\right|=0, \mid \Psi^{+}{ }_{B I_{1}}(x)-
$$

$\Psi^{+}{ }_{B I_{2}}(x)|=0,| \Gamma^{-}{ }_{B I_{1}}(x)-\Gamma^{-}{ }_{B I_{2}}(x)|=0,| \xi^{-}{ }_{B I_{1}}(x)-\xi^{-}{ }_{B I_{2}}(x) \mid=$

$0,\left|\Psi^{-}{ }_{B I_{1}}(x)-\Psi^{-}{ }_{B I_{2}}(x)\right|=0$, and $\cos (0)=1$.

Thus, $C s_{t}\left(B I_{1}, B I_{2}\right)=1, t=1,2,3$.

\section{Conversely}

$C s_{t}\left(B I_{1}, B I_{2}\right)=1$ then, 


$$
\begin{aligned}
& \left|\Gamma^{+}{ }_{B I_{1}}(x)-\Gamma^{+}{ }_{B I_{2}}(x)\right|=0,\left|\xi^{+}{ }_{B I_{1}}(x)-\xi^{+}{ }_{B I_{2}}(x)\right|=0, \mid \Psi^{+}{ }_{B I_{1}}(x)- \\
& \Psi^{+}{ }_{B I_{2}}(x)|=0,| \Gamma^{-}{ }_{B I_{1}}(x)-\Gamma^{-}{ }_{B I_{2}}(x)|=0,| \xi^{-}{ }_{B I_{1}}(x)-\xi^{-}{ }_{B I_{2}}(x) \mid= \\
& 0,\left|\Psi^{-}{ }_{B I_{1}}(x)-\Psi^{-}{ }_{B I_{2}}(x)\right|=0 .
\end{aligned}
$$

Also,

$$
\begin{aligned}
& \Gamma^{+}{ }_{B_{1}}(x)=\Gamma^{+}{ }_{B I_{2}}(x), \xi^{+}{ }_{B I_{1}}(x)=\xi^{+}{ }_{B I_{2}}(x), \Psi^{+}{ }_{B I_{1}}(x)=\Psi^{+}{ }_{B I_{2}}(x), \Gamma^{-}{ }_{B I_{1}}(x)= \\
& \Gamma^{-}{ }_{B I_{2}}(x), \xi^{-}{ }_{B I_{1}}(x)=\xi^{-}{ }_{B I_{2}}(x), \text { and } \Psi^{-}{ }_{B I_{1}}(x)=\Psi^{-}{ }_{B I_{2}}(x) .
\end{aligned}
$$

Then, $B I_{1}=B I_{2}$.

Example 1. Suppose that $B I_{1}=\langle 0.5,0.1,0.2,-0.1,-0.2,-0.1\rangle$ and $B I_{2}=$ $\langle 0.3,0.3,0.1,-0.2,-0.1,-0.15\rangle$ are two bipolar neutrosophic sets. Then, we have

$$
\begin{aligned}
& C s_{1}\left(B I_{1}, B I_{2}\right)=\cos \left(\frac{\pi}{12} \times(|0.5-0.3|+|0.1-0.3|+|0.2-0.1|+\mid-0.1+\right. \\
& 0.2|+|-0.2+0.1|+|-0.1+0.15 \mid))=0.981 \\
& \qquad S_{2}\left(B I_{1}, B I_{2}\right)=\cos \left(\frac{\pi}{4} \times \operatorname{Max}(|0.5-0.3|,|0.1-0.3|,|0.2-0.1|, \mid-0.1+\right. \\
& 0.2|,|-0.2+0.1|,|-0.1+0.15 \mid))=0.987 \\
& C s_{3}\left(B I_{1}, B I_{2}\right)=\cos \left(\frac{\pi}{2} \times\left(\frac{M_{1}+M_{2}}{2}\right)\right)=\cos \left(\frac{\pi}{2} \times\left(\frac{0.25}{2}\right)\right) 0.981 .
\end{aligned}
$$

Definition 2. Let $B I_{1}=$

$\left\{\left\langle x\left(\Gamma^{+}{ }_{B I_{1}}(x), \xi^{+}{ }_{B I_{1}}(x), \Psi^{+}{ }_{B I_{1}}(x), \Gamma^{-}{ }_{B I_{1}}(x), \xi^{-}{ }_{B I_{1}}(x), \Psi^{-}{ }_{B I_{1}}(x)\right)\right\rangle \mid x \in X\right\}$, and $B I_{2}=$ $\left\{\left\langle x\left(\Gamma^{+}{ }_{B I_{2}}(x), \xi^{+}{ }_{B I_{2}}(x), \Psi^{+}{ }_{B I_{2}}(x), \Gamma^{-}{ }_{B I_{2}}(x), \xi^{-}{ }_{B I_{2}}(x), \Psi^{-}{ }_{B I_{2}}(x)\right)\right\rangle \mid x \in X\right\}$ be two bipolar neutrosophic sets. Then, weighted cosine similarity measure among bipolar neutrosophic sets $B I_{1}$ and $B I_{2}$ denoted by $C s_{t}{ }^{w}\left(B I_{1}, B I_{2}\right), t=1,2,3$ is defined as follows:

$$
\begin{aligned}
& C S_{1}{ }^{w}\left(B I_{1}, B I_{2}\right)=w \times \cos \left(\frac{\pi}{12} \times\left(\left|\Gamma^{+}{ }_{B I_{1}}(x)-\Gamma^{+}{ }_{B I_{2}}(x)\right|+\left|\xi^{+}{ }_{B I_{1}}(x)-\xi^{+}{ }_{B I_{2}}(x)\right|+\right.\right. \\
& \left|\Psi^{+}{ }_{B I_{1}}(x)-\Psi^{+}{ }_{B I_{2}}(x)\right|+\left|\Gamma^{-}{ }_{B I_{1}}(x)-\Gamma^{-}{ }_{B I_{2}}(x)\right|+\left|\xi^{-}{ }_{B I_{1}}(x)-\xi^{-}{ }_{B I_{2}}(x)\right|+ \\
& \left.\left.\left|\Psi^{-}{ }_{B I_{1}}(x)-\Psi^{-}{ }_{B I_{2}}(x)\right|\right)\right) \\
& C S_{2}{ }^{w}\left(B I_{1}, B I_{2}\right)=w \times \cos \left(\frac{\pi}{4} \times \operatorname{Max}\left(\left|\Gamma^{+}{ }_{B I_{1}}(x)-\Gamma^{+}{ }_{B I_{2}}(x)\right|, \mid \xi^{+}{ }_{B I_{1}}(x)-\right.\right. \\
& \xi^{+}{ }_{B I_{2}}(x)|,| \Psi^{+}{ }_{B I_{1}}(x)-\Psi^{+}{ }_{B I_{2}}(x)|,| \Gamma^{-}{ }_{B I_{1}}(x)-\Gamma^{-}{ }_{B I_{2}}(x)|,| \xi^{-}{ }_{B I_{1}}(x)-\xi^{-}{ }_{B I_{2}}(x) \mid \\
& \left.\left.\left|\Psi^{-}{ }_{B I_{1}}(x)-\Psi^{-}{ }_{B I_{2}}(x)\right|\right)\right) \\
& C S_{3}{ }^{w}\left(B I_{1}, B I_{2}\right)=w \times \cos \left(\frac{\pi}{2} \times\left(\frac{M_{1}+M_{2}}{2}\right)\right)
\end{aligned}
$$

Since $w \in[0,1]$. 
The three types of weighted cosine similarity measures $C s_{t}{ }^{w}\left(B I_{1}, B I_{2}\right), t=1,2,3$ meet these characteristics:

a) $0 \leq C s_{t}{ }^{w}\left(B I_{1}, B I_{2}\right) \leq 1$,

b) $C s_{t}{ }^{w}\left(B I_{1}, B I_{2}\right)=C s_{t}{ }^{w}\left(B I_{2}, B I_{1}\right), t=1,2,3$,

c) $C s_{t}{ }^{w}\left(B I_{1}, B I_{2}\right)=1$, if and only if $B I_{1}=B I_{2}$.

\section{Proof}

The proof of properties for weighted cosine similarity measures of bipolar neutrosophic set is exactly as we have illustration of cosine similarity measures plus considering $w \in[0,1]$, thus it is not necessary to prove it again.

Example 2. Let $B I_{1}=\langle 0.5,0.1,0.2,-0.1,-0.2,-0.1\rangle$ and $B I_{2}=$ $\langle 0.3,0.3,0.1,-0.2,-0.1,-0.15\rangle$ are two bipolar neutrosophic sets and weight $w=0.5$.

Then, we have

$$
\begin{aligned}
& C s_{1}\left(B_{1}, B_{2}\right)=0.5 \times \cos \left(\frac{\pi}{12} \times(|0.5-0.3|+|0.1-0.3|+|0.2-0.1|+\mid-0.1+\right. \\
& 0.2|+|-0.2+0.1|+|-0.1+0.15 \mid))=0.490 . \\
& \quad C s_{2}\left(B_{1}, B_{2}\right)=0.5 \times \cos \left(\frac{\pi}{4} \times \operatorname{Max}(|0.5-0.3|,|0.1-0.3|,|0.2-0.1|, \mid-0.1+\right. \\
& 0.2|,|-0.2+0.1|,|-0.1+0.15 \mid))=0.493 . \\
& C s_{3}\left(B_{1}, B_{2}\right)=0.5 \times \cos \left(\frac{\pi}{2} \times\left(\frac{M_{1}+M_{2}}{2}\right)\right)=0.490 .
\end{aligned}
$$

Definition 3. Let $I V_{1}=$

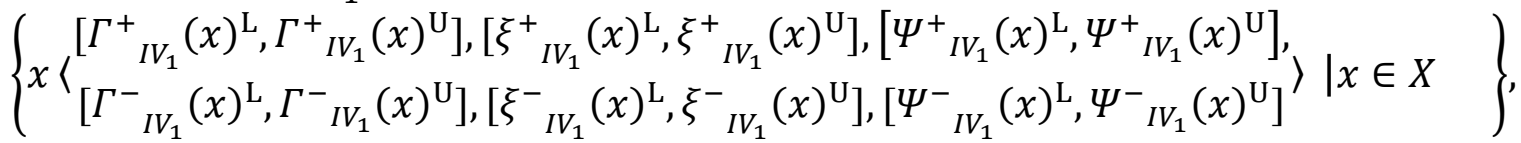
and $I V_{2}=$

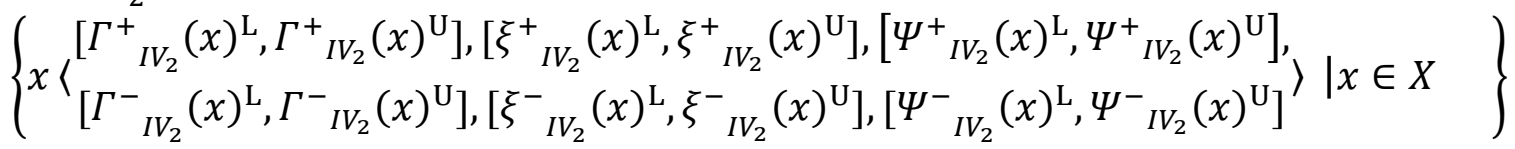

be two interval valued bipolar neutrosophic sets. Then, cosine similarity measure among bipolar neutrosophic sets $I V_{1}$ and $I V_{2}$ denoted by $C s_{t}\left(I V_{1}, I V_{2}\right), t=1,2,3$ is defined as follows:

$$
\begin{aligned}
& C S_{1}\left(I V_{1}, I V_{2}\right)=\cos \left(\frac{\pi}{24} \times\left(\left|\Gamma^{+}{ }_{I V_{1}}(x)^{\mathrm{L}}-\Gamma^{+}{ }_{I V_{2}}(x)^{\mathrm{L}}\right|+\left|\Gamma^{+}{ }_{I V_{1}}(x)^{\mathrm{U}}-\Gamma^{+}{ }_{I V_{2}}(x)^{\mathrm{U}}\right|+\right.\right. \\
& \left|\xi^{+}{ }_{I V_{1}}(x)^{\mathrm{L}}-\xi^{+}{ }_{I V_{2}}(x)^{\mathrm{L}}\right|+\left|\xi^{+}{ }_{I V_{1}}(x)^{\mathrm{U}}-\xi^{+}{ }_{I V_{2}}(x)^{\mathrm{U}}\right|+\left|{\Psi^{+}}{ }_{I V_{1}}(x)^{\mathrm{L}}-\Psi^{+}{ }_{I V_{2}}(x)^{\mathrm{L}}\right|+ \\
& \left|\Psi^{+}{ }_{{ }_{I V}}(x)^{\mathrm{U}}-\Psi^{+}{ }_{I V_{2}}(x)^{\mathrm{U}}\right|+\left|\Gamma^{-}{ }_{I V_{1}}(x)^{\mathrm{L}}-\Gamma^{-}{ }_{I V_{2}}(x)^{\mathrm{L}}\right|+\left|\Gamma^{-}{ }_{I V_{1}}(x)^{\mathrm{U}}-\Gamma^{-}{ }_{I V_{2}}(x)^{\mathrm{U}}\right|+
\end{aligned}
$$




$$
\begin{aligned}
& \left|\xi^{-}{ }_{I V_{1}}(x)^{\mathrm{L}}-\xi^{-}{ }_{I V_{2}}(x)^{\mathrm{L}}\right|+\left|\xi^{-}{ }_{I V_{1}}(x)^{\mathrm{U}}-\xi^{-}{ }_{I V_{2}}(x)^{\mathrm{U}}\right|+\quad\left|\Psi^{-}{ }_{I V_{1}}(x)^{\mathrm{L}}-\Psi^{-}{ }_{I V_{2}}(x)^{\mathrm{L}}\right|+ \\
& \left.\left.\left|\Psi^{-}{ }_{I V_{1}}(x)^{\mathrm{U}}-\Psi^{-}{ }_{I V_{2}}(x)^{\mathrm{U}}\right|\right)\right)
\end{aligned}
$$

$$
\begin{aligned}
& C S_{2}\left(I V_{1}, I V_{2}\right)=\cos \left(\frac{\pi}{4} \times \operatorname{Max}\left(\left|\Gamma^{+}{ }_{I V_{1}}(x)^{\mathrm{L}}-\Gamma^{+}{ }_{I V_{2}}(x)^{\mathrm{L}}\right|,\left|\Gamma^{+}{ }_{I V_{1}}(x)^{\mathrm{U}}-\Gamma^{+}{ }_{I V_{2}}(x)^{\mathrm{U}}\right|\right.\right. \\
& \left|\xi^{+}{ }_{I V_{1}}(x)^{\mathrm{L}}-\xi^{+}{ }_{I V_{2}}(x)^{\mathrm{L}}\right|, \quad\left|\xi^{+}{ }_{I V_{1}}(x)^{\mathrm{U}}-\xi^{+}{ }_{I V_{2}}(x)^{\mathrm{U}}\right|,\left|\Psi^{+}{ }_{I V_{1}}(x)^{\mathrm{L}}-\Psi^{+}{ }_{I V_{2}}(x)^{\mathrm{L}}\right| \\
& \left|\Psi^{+}{ }_{I V_{1}}(x)^{\mathrm{U}}-\Psi^{+}{ }_{I V_{2}}(x)^{\mathrm{U}}\right|,\left|\Gamma^{-}{ }_{I V_{1}}(x)^{\mathrm{L}}-\Gamma^{-}{ }_{I V_{2}}(x)^{\mathrm{L}}\right|,\left|\Gamma^{-}{ }_{I V_{1}}(x)^{\mathrm{U}}-\Gamma^{-}{ }_{I V_{2}}(x)^{\mathrm{U}}\right| \\
& \left|\xi^{-}{ }_{I V_{1}}(x)^{\mathrm{L}}-\xi^{-}{ }_{I V_{2}}(x)^{\mathrm{L}}\right|,\left|\xi^{-}{ }_{I V_{1}}(x)^{\mathrm{U}}-\xi^{-}{ }_{I V_{2}}(x)^{\mathrm{U}}\right|,\left|\Psi^{-}{ }_{I V_{1}}(x)^{\mathrm{L}}-\Psi^{-}{ }_{I V_{2}}(x)^{\mathrm{L}}\right| \\
& \left.\left.\left|\Psi^{-}{ }_{I V_{1}}(x)^{\mathrm{U}}-\Psi^{-}{ }_{I V_{2}}(x)^{\mathrm{U}}\right|\right)\right) \\
& C_{3}\left(I V_{1}, I V_{2}\right)=\cos \left(\frac{\pi}{2} \times\left(\frac{M_{1}+M_{2}}{2}\right)\right)
\end{aligned}
$$

Since, $M_{1}=$

$\operatorname{Max}\left(\left|\Gamma^{+}{ }_{I V_{1}}(x)^{\mathrm{L}}-\Gamma^{+}{ }_{I V_{2}}(x)^{\mathrm{L}}\right|,\left|\Gamma^{+}{ }_{I V_{1}}(x)^{\mathrm{U}}-\Gamma^{+}{ }_{I V_{2}}(x)^{\mathrm{U}}\right|,\left|\xi^{+}{ }_{I V_{1}}(x)^{\mathrm{L}}-\xi^{+}{ }_{I V_{2}}(x)^{\mathrm{L}}\right|\right.$,

$\left|\xi^{+}{ }_{I V_{1}}(x)^{\mathrm{U}}-\xi^{+}{ }_{I V_{2}}(x)^{\mathrm{U}}\right|, \quad\left|\Psi^{+}{ }_{I V_{1}}(x)^{\mathrm{L}}-\Psi^{+}{ }_{I V_{2}}(x)^{\mathrm{L}}\right|,\left|\Psi^{+}{ }_{I V_{1}}(x)^{\mathrm{U}}-\Psi^{+}{ }_{I V_{2}}(x)^{\mathrm{U}}\right|$

$\left|\Gamma^{-}{ }_{I V_{1}}(x)^{\mathrm{L}}-\Gamma^{-}{ }_{I V_{2}}(x)^{\mathrm{L}}\right|, \quad\left|\Gamma^{-}{ }_{I V_{1}}(x)^{\mathrm{U}}-\Gamma^{-}{ }_{I V_{2}}(x)^{\mathrm{U}}\right|,\left|\xi^{-}{ }_{I V_{1}}(x)^{\mathrm{L}}-\xi^{-}{ }_{I V_{2}}(x)^{\mathrm{L}}\right|$,

$\left.\left|\xi^{-}{ }_{I V_{1}}(x)^{\mathrm{U}}-\xi^{-}{ }_{I V_{2}}(x)^{\mathrm{U}}\right|,\left|\Psi^{-}{ }_{I V_{1}}(x)^{\mathrm{L}}-\Psi^{-}{ }_{I V_{2}}(x)^{\mathrm{L}}\right|,\left|\Psi^{-}{ }_{I V_{1}}(x)^{\mathrm{U}}-\Psi^{-}{ }_{I V_{2}}(x)^{\mathrm{U}}\right|\right)$,

and $M_{2}=$

$\operatorname{Min}\left(\left|\Gamma^{+}{ }_{I V_{1}}(x)^{\mathrm{L}}-\Gamma^{+}{ }_{I V_{2}}(x)^{\mathrm{L}}\right|,\left|\Gamma^{+}{ }_{I V_{1}}(x)^{\mathrm{U}}-\Gamma^{+}{ }_{I V_{2}}(x)^{\mathrm{U}}\right|,\left|\xi^{+}{ }_{I V_{1}}(x)^{\mathrm{L}}-\xi^{+}{ }_{I V_{2}}(x)^{\mathrm{L}}\right|\right.$,

$\left|\xi^{+}{ }_{I V_{1}}(x)^{\mathrm{U}}-\xi^{+}{ }_{I V_{2}}(x)^{\mathrm{U}}\right|,\left|\Psi^{+}{ }_{I V_{1}}(x)^{\mathrm{L}}-\Psi^{+}{ }_{I V_{2}}(x)^{\mathrm{L}}\right|,\left|\Psi^{+}{ }_{I V_{1}}(x)^{\mathrm{U}}-\Psi^{+}{ }_{I V_{2}}(x)^{\mathrm{U}}\right|$,

$\left|\Gamma^{-}{ }_{I V_{1}}(x)^{\mathrm{L}}-\Gamma^{-}{ }_{I V_{2}}(x)^{\mathrm{L}}\right|,\left|\Gamma^{-}{ }_{I V_{1}}(x)^{\mathrm{U}}-\Gamma^{-}{ }_{I V_{2}}(x)^{\mathrm{U}}\right|,\left|\xi^{-}{ }_{I V_{1}}(x)^{\mathrm{L}}-\xi^{-}{ }_{I V_{2}}(x)^{\mathrm{L}}\right|$,

$\left.\left|\xi^{-}{ }_{I V_{1}}(x)^{\mathrm{U}}-\xi^{-}{ }_{I V_{2}}(x)^{\mathrm{U}}\right|,\left|\Psi^{-}{ }_{I V_{1}}(x)^{\mathrm{L}}-\Psi^{-}{ }_{I V_{2}}(x)^{\mathrm{L}}\right|,\left|\Psi^{-}{ }_{I V_{1}}(x)^{\mathrm{U}}-\Psi^{-}{ }_{I V_{2}}(x)^{\mathrm{U}}\right|\right)$.

The three measures of similarity for IVBNSs $C s_{t}\left(I V_{1}, I V_{2}\right), t=1,2,3$ meet these characteristics:

a) $0 \leq C s_{t}\left(I V_{1}, I V_{2}\right) \leq 1$,

\section{Proof}

For any interval valued bipolar neutrosophic set , $\Gamma^{+}{ }_{I V}(x)^{\mathrm{L}}, \Gamma^{+}{ }_{I V}(x)^{\mathrm{U}}, \xi^{+}{ }_{I V}(x)^{\mathrm{L}}, \xi^{+}{ }_{I V}(x)^{\mathrm{U}}$ and $\Psi^{+}{ }_{I V}(x)^{\mathrm{L}}, \Psi^{+}{ }_{I V}(x)^{\mathrm{U}}$ lie within $[0,1]$ and $\Gamma^{-}{ }_{I V}(x)^{\mathrm{L}}, \Gamma^{-}{ }_{I V}(x)^{\mathrm{U}}, \xi^{-}{ }_{I V}(x)^{\mathrm{L}}, \xi^{-}{ }_{I V}(x)^{\mathrm{U}}$ and $\Psi^{-}{ }_{I V}(x)^{\mathrm{L}}, \Psi^{-}{ }_{I V}(x)^{\mathrm{U}}$ lie within $[-1,0]$. So, the values of the suggested cosine functions are within $[0,1]$. Hence, $0 \leq C s_{t}\left(I V_{1}, I V_{2}\right) \leq 1$,

b) $C s_{t}\left(I V_{1}, I V_{2}\right)=C s_{t}\left(I V_{2}, I V_{1}\right), t=1,2,3$.

\section{Proof}

Since,

$$
\left|\Gamma^{+}{ }_{I V_{1}}(x)^{L}-\Gamma^{+}{ }_{I V_{2}}(x)^{L}\right|=\left|\Gamma^{+}{ }_{I V_{2}}(x)^{L}-\Gamma^{+}{ }_{I V_{1}}(x)^{L}\right|,\left|\Gamma^{+}{ }_{I V_{1}}(x)^{\mathrm{U}}-\Gamma^{+}{ }_{I V_{2}}(x)^{\mathrm{U}}\right|
$$




$$
\begin{aligned}
& =\left|\Gamma^{+}{ }_{I V_{2}}(x)^{\mathrm{U}}-\Gamma^{+}{ }_{I V_{1}}(x)^{\mathrm{U}}\right|,\left|\xi^{+}{ }_{I V_{1}}(x)^{\mathrm{L}}-\xi^{+}{ }_{I V_{2}}(x)^{\mathrm{L}}\right|=\mid \xi^{+}{ }_{I V_{2}}(x)^{\mathrm{L}}- \\
& \xi^{+}{ }_{I V_{1}}(x)^{\mathrm{L}}|,| \xi^{+}{ }_{I V_{1}}(x)^{\mathrm{U}}-\xi^{+}{ }_{I V_{2}}(x)^{\mathrm{U}}|| \xi^{+}{ }_{I V_{2}}(x)^{\mathrm{U}}-\xi^{+}{ }_{I V_{1}}(x)^{\mathrm{U}}|,| \Psi^{+}{ }_{I V_{1}}(x)^{\mathrm{L}}- \\
& {\Psi^{+}}^{{ }_{I V_{2}}}(x)^{\mathrm{L}}|=| \Psi^{+}{ }_{I V_{2}}(x)^{\mathrm{L}}-\Psi^{+}{ }_{I V_{1}}(x)^{\mathrm{L}}|,| \Psi^{+}{ }_{I V_{1}}(x)^{\mathrm{U}}-\Psi^{+}{ }_{I V_{2}}(x)^{\mathrm{U}} \mid= \\
& \left|\Psi^{+}{ }_{I V_{2}}(x)^{\mathrm{U}}-\Psi^{+}{ }_{I V_{1}}(x)^{\mathrm{U}}\right|,\left|\Gamma^{-}{ }_{I V_{1}}(x)^{L}-\Gamma^{-}{ }_{I V_{2}}(x)^{L}\right|=\left|\Gamma^{-}{ }_{I V_{2}}(x)^{L}-\Gamma^{-}{ }_{I V_{1}}(x)^{L}\right| \\
& ,\left|\Gamma^{-}{ }_{I V_{1}}(x)^{\mathrm{U}}-\Gamma^{-}{ }_{I V_{2}}(x)^{\mathrm{U}}\right|=\left|\Gamma^{-}{ }_{I V_{2}}(x)^{\mathrm{U}}-\Gamma^{-}{ }_{I V_{1}}(x)^{\mathrm{U}}\right|, \mid \xi^{-}{ }_{I V_{1}}(x)^{\mathrm{L}}- \\
& \xi^{-}{ }_{I V_{2}}(x)^{\mathrm{L}}|=| \xi^{-}{ }_{I V_{2}}(x)^{\mathrm{L}}-\xi^{-}{ }_{I V_{1}}(x)^{\mathrm{L}}|,| \xi^{-}{ }_{I V_{1}}(x)^{\mathrm{U}}-\xi^{-}{ }_{I V_{2}}(x)^{\mathrm{U}} \mid= \\
& \left|\xi^{-}{ }_{I V_{2}}(x)^{\mathrm{U}}-\xi^{-}{ }_{I V_{1}}(x)^{\mathrm{U}}\right|,\left|\Psi^{-}{ }_{I V_{1}}(x)^{\mathrm{L}}-{\Psi^{-}}_{I V_{2}}(x)^{\mathrm{L}}\right|=\mid \Psi^{-}{ }_{I V_{2}}(x)^{\mathrm{L}}- \\
& \Psi^{-}{ }_{I V_{1}}(x)^{\mathrm{L}}|,| \Psi^{-}{ }_{I V_{1}}(x)^{\mathrm{U}}-\Psi^{-}{ }_{I V_{2}}(x)^{\mathrm{U}}|=| \Psi^{-}{ }_{I V_{2}}(x)^{\mathrm{U}}-\Psi^{-}{ }_{I V_{1}}(x)^{\mathrm{U}} \mid \text {. }
\end{aligned}
$$

Thus, $C s_{t}\left(I V_{1}, I V_{2}\right)=C s_{t}\left(I V_{2}, I V_{1}\right), t=1,2,3$.

c) $C s_{t}\left(I V_{1}, I V_{2}\right)=1$, if and only if $I V_{1}=I V_{2}$.

\section{Proof}

For any two interval valued bipolar neutrosophic sets $I V_{1}$ and $I V_{2}$, when $I V_{1}=I V_{2}$ then,

$$
\begin{aligned}
& \Gamma^{+}{ }_{I V_{1}}(x)^{L}=\Gamma^{+}{ }_{I V_{2}}(x)^{L}, \Gamma^{+}{ }_{I V_{1}}(x)^{U}=\Gamma^{+}{ }_{I V_{2}}(x)^{U}, \xi^{+}{ }_{I V_{1}}(x)^{L}=\xi^{+}{ }_{I V_{2}}(x)^{L}, \\
& \xi^{+}{ }_{I V_{1}}(x)^{U}=\xi^{+}{ }_{I V_{2}}(x)^{U}, \Psi^{+}{ }_{I V_{1}}(x)^{L}=\Psi^{+}{ }_{I V_{2}}(x)^{L}, \Psi^{+}{ }_{I V_{1}}(x)^{U}=\Psi^{+}{ }_{I V_{2}}(x)^{U} \\
& { }^{-} \Gamma^{-}{ }_{I V_{1}}(x)^{L}=\Gamma^{-}{ }_{I V_{2}}(x)^{L}, \Gamma^{-}{ }_{I V_{1}}(x)^{U}=\Gamma^{-}{ }_{I V_{2}}(x)^{U}, \xi^{-}{ }_{I V_{1}}(x)^{L}=\xi^{-}{ }_{I V_{2}}(x)^{L} \\
& { }^{\xi^{-}}{ }_{I V_{1}}(x)^{U}=\xi^{-}{ }_{I V_{2}}(x)^{U}, \Psi^{-}{ }_{I V_{1}}(x)^{L}=\Psi^{-}{ }_{I V_{2}}(x)^{L}, \Psi^{-}{ }_{I V_{1}}(x)^{U}=\Psi^{-}{ }_{I V_{2}}(x)^{U}
\end{aligned}
$$

thus,

$$
\begin{aligned}
& \left|\Gamma^{+}{ }_{I V_{1}}(x)^{\mathrm{L}}-\Gamma^{+}{ }_{I V_{2}}(x)^{\mathrm{L}}\right|=0,\left|\Gamma^{+}{ }_{I V_{1}}(x)^{\mathrm{U}}-\Gamma^{+}{ }_{I V_{2}}(x)^{\mathrm{U}}\right|=0, \mid \xi^{+}{ }_{I V_{1}}(x)^{\mathrm{L}}- \\
& \xi^{+}{ }_{I V_{2}}(x)^{\mathrm{L}}|=0,| \xi^{+}{ }_{I V_{1}}(x)^{\mathrm{U}}-\xi^{+}{ }_{I V_{2}}(x)^{\mathrm{U}}|=0,| \Psi^{+}{ }_{I V_{1}}(x)^{\mathrm{L}}-\Psi^{+}{ }_{I V_{2}}(x)^{\mathrm{L}} \mid=0 \text {, } \\
& \left|\Psi^{+}{ }_{I V_{1}}(x)^{\mathrm{U}}-\Psi^{+}{ }_{I V_{2}}(x)^{\mathrm{U}}\right|=0, \quad\left|\Gamma^{-}{ }_{I V_{1}}(x)^{\mathrm{L}}-\Gamma^{-}{ }_{I V_{2}}(x)^{\mathrm{L}}\right|=0, \mid \Gamma^{-}{ }_{I V_{1}}(x)^{\mathrm{U}}- \\
& \Gamma^{-}{ }_{I V_{2}}(x)^{\mathrm{U}}|=0, \quad| \xi^{-}{ }_{I V_{1}}(x)^{\mathrm{L}}-\xi^{-}{ }_{I V_{2}}(x)^{\mathrm{L}}|=0, \quad| \xi^{-}{ }_{I V_{1}}(x)^{\mathrm{U}}-\xi^{-}{ }_{I V_{2}}(x)^{\mathrm{U}} \mid= \\
& 0,\left|\Psi^{-}{ }_{I V_{1}}(x)^{\mathrm{L}}-\Psi^{-}{ }_{I V_{2}}(x)^{\mathrm{L}}\right|=0,\left|\Psi^{-}{ }_{I V_{1}}(x)^{\mathrm{U}}-\Psi^{-}{ }_{I V_{2}}(x)^{\mathrm{U}}\right|=0 \text {, and } \cos (0)= \\
& \text { 1. Thus, } C s_{t}\left(I V_{1}, I V_{2}\right)=1, t=1,2,3 \text {. }
\end{aligned}
$$

\section{Conversely}

$C s_{t}\left(I V_{1}, I V_{2}\right)=1$ then,

$$
\begin{aligned}
& \left|\Gamma^{+}{ }_{I V_{1}}(x)^{\mathrm{L}}-\Gamma^{+}{ }_{I V_{2}}(x)^{\mathrm{L}}\right|=0,\left|\Gamma^{+}{ }_{I V_{1}}(x)^{\mathrm{U}}-\Gamma^{+}{ }_{I V_{2}}(x)^{\mathrm{U}}\right|=0, \mid \xi^{+}{ }_{I V_{1}}(x)^{\mathrm{L}}- \\
& \xi^{+}{ }_{I V_{2}}(x)^{\mathrm{L}}|=0,| \xi^{+}{ }_{I V_{1}}(x)^{\mathrm{U}}-\xi^{+}{ }_{I V_{2}}(x)^{\mathrm{U}}|=0,| \Psi^{+}{ }_{I V_{1}}(x)^{\mathrm{L}}-\Psi^{+}{ }_{I V_{2}}(x)^{\mathrm{L}} \mid= \\
& 0,\left|\Psi^{+}{ }_{I V_{1}}(x)^{\mathrm{U}}-\Psi^{+}{ }_{I V_{2}}(x)^{\mathrm{U}}\right|=0,\left|\Gamma^{-}{ }_{I V_{1}}(x)^{\mathrm{L}}-\Gamma^{-}{ }_{I V_{2}}(x)^{\mathrm{L}}\right|=0, \quad \mid \Gamma^{-}{ }_{I V_{1}}(x)^{\mathrm{U}}- \\
& \Gamma^{-}{ }_{I V_{2}}(x)^{\mathrm{U}}|=0,| \xi^{-}{ }_{I V_{1}}(x)^{\mathrm{L}}-\xi^{-}{ }_{I V_{2}}(x)^{\mathrm{L}}|=0,| \xi^{-}{ }_{I V_{1}}(x)^{\mathrm{U}}-\xi^{-}{ }_{I V_{2}}(x)^{\mathrm{U}} \mid=0 \text {, } \\
& \left|\Psi^{-}{ }_{I V_{1}}(x)^{\mathrm{L}}-\Psi^{-}{ }_{I V_{2}}(x)^{\mathrm{L}}\right|=0,\left|\Psi^{-}{ }_{I V_{1}}(x)^{\mathrm{U}}-\Psi_{{ }_{I V_{2}}}(x)^{\mathrm{U}}\right|=0 \text { and then, } \\
& \Gamma^{+}{ }_{I V_{1}}(x)^{L}=\Gamma^{+}{ }_{I V_{2}}(x)^{L}, \Gamma^{+}{ }_{I V_{1}}(x)^{U}=\Gamma^{+}{ }_{I V_{2}}(x)^{U}, \xi^{+}{ }_{I V_{1}}(x)^{L}=\xi^{+}{ }_{I V_{2}}(x)^{L} \text {, } \\
& \xi^{+}{ }_{I V_{1}}(x)^{U}=\xi^{+}{ }_{I V_{2}}(x)^{U}, \Psi^{+}{ }_{I V_{1}}(x)^{L}=\Psi^{+}{ }_{I V_{2}}(x)^{L}, \Psi^{+}{ }_{I V_{1}}(x)^{U}=\Psi^{+}{ }_{I V_{2}}(x)^{U}
\end{aligned}
$$




$$
\begin{aligned}
& { }^{,} \Gamma^{-}{ }_{I V_{1}}(x)^{L}=\Gamma^{-}{ }_{I V_{2}}(x)^{L}, \Gamma^{-}{ }_{I V_{1}}(x)^{U}=\Gamma^{-}{ }_{I V_{2}}(x)^{U}, \xi^{-}{ }_{I V_{1}}(x)^{L}=\xi^{-}{ }_{I V_{2}}(x)^{L} \\
& { }^{-} \xi^{-}{ }_{I V_{1}}(x)^{U}=\xi^{-}{ }_{I V_{2}}(x)^{U}, \Psi^{-}{ }_{I V_{1}}(x)^{L}=\Psi^{-}{ }_{I V_{2}}(x)^{L}, \Psi^{-}{ }_{I V_{1}}(x)^{U}=\Psi^{-}{ }_{I V_{2}}(x)^{U}, \\
& \text { thus, } I V_{1}=I V_{2} .
\end{aligned}
$$

Example 3. Suppose that $I V_{1}=$

$\langle[0.5,0.7],[0.1,0.3],[0.2,0.4],[-0.2,-0.1],[-0.3,-0.1],[-0.4,-0.2]\rangle$ and

$I V_{2}=\langle[0.4,0.6],[0.1,0.2],[0.1,0.3],[-0.3,-0.1],[-0.4,-0.2],[-0.5,-0.3]\rangle$ are

two interval valued bipolar neutrosophic sets. Then, we have

$$
\begin{aligned}
& C s_{1}\left(I V_{1}, I V_{2}\right)=\cos \left(\frac{\pi}{24} \times(|0.5-0.4|+|0.7-0.6|+|0.1-0.1|+\mid 0.3-\right. \\
& 0.2|+| 0.2-0.1|+| 0.4-0.3|+|-0.2+0.3|+|-0.1+0.1|+|-0.3+0.4 \mid+ \\
& |-0.1+0.2|+|-0.4+0.5|+|-0.2+0.3|))=0.991 \\
& C s_{2}\left(I V_{1}, I V_{2}\right)=\cos \left(\frac{\pi}{4} \times \max (|0.5-0.4|,|0.7-0.6|,|0.1-0.1|,|0.3-0.2|, \mid 0.2-\right. \\
& 0.1|,| 0.4-0.3|,|-0.2+0.3|,|-0.1+0.1|,|-0.3+0.4|,|-0.1+0.2|,|-0.4+ \\
& 0.5|,|-0.2+0.3 \mid))=0.996 \\
& C s_{3}\left(I V_{1}, I V_{2}\right)=\cos \left(\frac{\pi}{2} \times\left(\frac{0.1}{2}\right)\right)=0.996
\end{aligned}
$$

Definition 4. The weighted cosine similarity measures among two IVBNSs $I V_{1}$ and $I V_{2}$ denoted by $C s_{t}{ }^{w}\left(I V_{1}, I V_{2}\right), t=1,2,3$ are defined as follows:

$$
\begin{aligned}
& C_{1}{ }^{w}\left(I V_{1}, I V_{2}\right)=\quad w \times \cos \left(\frac{\pi}{24} \times\left(\left|\Gamma^{+}{ }_{I V_{1}}(x)^{\mathrm{L}}-\Gamma^{+}{ }_{I V_{2}}(x)^{\mathrm{L}}\right|+\mid \Gamma^{+}{ }_{I V_{1}}(x)^{\mathrm{U}}-\right.\right. \\
& \Gamma^{+}{ }_{I V_{2}}(x)^{\mathrm{U}}|+| \xi^{+}{ }_{I V_{1}}(x)^{\mathrm{L}}-\xi^{+}{ }_{I V_{2}}(x)^{\mathrm{L}}|+| \xi^{+}{ }_{I V_{1}}(x)^{\mathrm{U}}-\xi^{+}{ }_{I V_{2}}(x)^{\mathrm{U}}|+| \Psi^{+}{ }_{I V_{1}}(x)^{\mathrm{L}}- \\
& \Psi^{+}{ }_{I V_{2}}(x)^{\mathrm{L}}|+| \Psi^{+}{ }_{I V_{1}}(x)^{\mathrm{U}}-\Psi^{+}{ }_{I V_{2}}(x)^{\mathrm{U}}|+| \Gamma^{-}{ }_{I V_{1}}(x)^{\mathrm{L}}-\Gamma^{-}{ }_{I V_{2}}(x)^{\mathrm{L}}|+| \Gamma^{-}{ }_{I V_{1}}(x)^{\mathrm{U}}- \\
& \Gamma^{-}{ }_{I V_{2}}(x)^{\mathrm{U}}|+| \xi^{-}{ }_{I V_{1}}(x)^{\mathrm{L}}-\xi^{-}{ }_{I V_{2}}(x)^{\mathrm{L}}|+| \xi^{-}{ }_{I V_{1}}(x)^{\mathrm{U}}-\xi^{-}{ }_{I V_{2}}(x)^{\mathrm{U}}|+| \Psi^{-}{ }_{I V_{1}}(x)^{\mathrm{L}}- \\
& \left.\left.\Psi^{-}{ }_{I V_{2}}(x)^{\mathrm{L}}|+| \Psi^{-}{ }_{I V_{1}}(x)^{\mathrm{U}}-\Psi^{-}{ }_{I V_{2}}(x)^{\mathrm{U}} \mid\right)\right) \\
& C s_{2}{ }^{w}\left(I V_{1}, I V_{2}\right)=w \times \cos \left(\frac{\pi}{4} \times \operatorname{Max}\left(\left|\Gamma^{+}{ }_{I V_{1}}(x)^{\mathrm{L}}-\Gamma^{+}{ }_{I V_{2}}(x)^{\mathrm{L}}\right|, \mid \Gamma^{+}{ }_{I V_{1}}(x)^{\mathrm{U}}-\right.\right. \\
& \Gamma^{+}{ }_{I V_{2}}(x)^{\mathrm{U}}|,| \xi^{+}{ }_{I V_{1}}(x)^{\mathrm{L}}-\xi^{+}{ }_{I V_{2}}(x)^{\mathrm{L}}|,| \xi^{+}{ }_{I V_{1}}(x)^{\mathrm{U}}-\xi^{+}{ }_{I V_{2}}(x)^{\mathrm{U}}|,| \Psi^{+}{ }_{I V_{1}}(x)^{\mathrm{L}}- \\
& \Psi^{+}{ }_{I V_{2}}(x)^{\mathrm{L}}|,| \Psi^{+}{ }_{I V_{1}}(x)^{\mathrm{U}}-\Psi^{+}{ }_{I V_{2}}(x)^{\mathrm{U}}|,| \Gamma^{-}{ }_{I V_{1}}(x)^{\mathrm{L}}-\Gamma^{-}{ }_{I V_{2}}(x)^{\mathrm{L}}|,| \Gamma^{-}{ }_{I V_{1}}(x)^{\mathrm{U}}- \\
& \Gamma_{I V_{2}}^{-}(x)^{\mathrm{U}}|,| \xi^{-}{ }_{I V_{1}}(x)^{\mathrm{L}}-\xi^{-}{ }_{I V_{2}}(x)^{\mathrm{L}}|,| \xi^{-}{ }_{I V_{1}}(x)^{\mathrm{U}}-\xi^{-}{ }_{I V_{2}}(x)^{\mathrm{U}}|,| \Psi^{-}{ }_{I V_{1}}(x)^{\mathrm{L}}- \\
& \left.\left.\Psi^{-}{ }_{I V_{2}}(x)^{\mathrm{L}}|,| \Psi^{-}{ }_{I V_{1}}(x)^{\mathrm{U}}-\Psi^{-}{ }_{I V_{2}}(x)^{\mathrm{U}} \mid\right)\right) \\
& C s_{3}{ }^{w}\left(I V_{1}, I V_{2}\right)=w \times \cos \left(\frac{\pi}{2} \times\left(\frac{M_{1}+M_{2}}{2}\right)\right)
\end{aligned}
$$

Since $w$ belongs to $[0,1]$ and $\sum w=1$.

The weighted bipolar neutrosophic cosine similarity measure $C s_{t}{ }^{w}\left(I V_{1}, I V_{2}\right), t=1,2,3$, meet these characteristics:
a) $0 \leq C s_{t}{ }^{w}\left(I V_{1}, I V_{2}\right) \leq 1$, 
b) $C s_{t}{ }^{w}\left(I V_{1}, I V_{2}\right)=C s_{t}{ }^{w}\left(I V_{2}, I V_{1}\right), t=1,2,3$.

c) $C_{t}{ }^{w}\left(I V_{1}, I V_{2}\right)=1$, if and only if $I V_{1}=I V_{2}$.

\section{Proof}

The proof of properties for weighted cosine similarity measures of interval valued bipolar neutrosophic set is similar of cosine similarity measures for interval bipolar neutrosophic set plus considering $w \in[0,1]$.

Example 4. Suppose that

$I V_{1}=\langle[0.5,0.7],[0.1,0.3],[0.2,0.4],[-0.2,-0.1],[-0.3,-0.1],[-0.4,-0.2]\rangle$ and $I V_{2}=\langle[0.4,0.6],[0.1,0.2],[0.1,0.3],[-0.3,-0.1],[-0.4,-0.2],[-0.5,-0.3]\rangle$ are two interval valued bipolar neutrosophic sets and $w=0.5$. Then, we have $C_{1}{ }^{w}\left(I V_{1}, I V_{2}\right)=0.5 \times \cos \left(\frac{\pi}{24} \times(|0.5-0.4|+|0.7-0.6|+|0.1-0.1|+\mid 0.3-\right.$ $0.2|+| 0.2-0.1|+| 0.4-0.3|+|-0.2+0.3|+|-0.1+0.1|+|-0.3+0.4 \mid+$ $|-0.1+0.2|+|-0.4+0.5|+|-0.2+0.3|))=0.495$.

$C s_{2}{ }^{w}\left(I V_{1}, I V_{2}\right)=0.5 \times \cos \left(\frac{\pi}{4} \times \max (|0.5-0.4|,|0.7-0.6|,|0.1-0.1|, \mid 0.3-\right.$ $0.2|| 0.2-,0.1|| 0.4-,0.3||-0.2+,0.3||-0.1+,0.1||-0.3+,0.4||-0.1+$, $0.2||-0.4+,0.5||-0.2+0.3 \mid),)=0.498$.

$C s_{3}{ }^{w}\left(I V_{1}, I V_{2}\right)=0.5 \times \cos \left(\frac{\pi}{2} \times\left(\frac{0.1}{2}\right)\right)=0.498$.

\subsection{Comparison with other measures of similarity}

In this part we compared proposed measures of similarity with other presented measures in literature to demonstrate the superiority of proposed measures.

Here, four dissimilar patterns, state 1 to state 4 are presented and the results obtained by Eqs. (1-4), and (7-9) are listed in Table 1. From Table 1, it is seen that the suggested measures of similarity can overcome the deficiencies of producing unreasonable or undefined results. Also, there is a clear contradiction between proposed measures of similarity in $[42,43]$ and their presented properties. Indeed, our proposed measures appears stronger differentiation among them.

\begin{tabular}{ccccc}
\hline & State 1 & State 2 & State 3 & State 4 \\
\hline $\boldsymbol{E}$ & $\langle(1,1,0,-1,-1,0)\rangle$ & $\langle(1,1,0,-1,-1,0)\rangle$ & $\langle(1,0,0,-1,0,0)\rangle$ & $\langle(0,0,0,-1,0,0)\rangle$ \\
$\boldsymbol{F}$ & $\langle(0,1,1,-1,-1,0)\rangle$ & $\langle(1,1,0,-1,-1,0)\rangle$ & $\langle(1,0,0,-1,0,0)\rangle$ & $\langle(0,0,0,-1,0,0)\rangle$ \\
Eq.(1) [43] & Null & 0 & 0 & 0.5 \\
Eq.(2) [43] & Null & 0 & 0 & 0.5 at $w=1$ \\
Eq.(3) [43] & Null & Null & 0 & 0.07 at $\lambda=0.1$ \\
Eq.(4) $[$ 42] & -0.1 & 0 & 0 & -0.5 \\
$\boldsymbol{C s}_{\mathbf{1}}(\boldsymbol{E}, \boldsymbol{F})$ & 0.86 & 1 & 1 & 1 \\
$\boldsymbol{C s}_{\mathbf{2}}(\boldsymbol{E}, \boldsymbol{F})$ & 0.71 & 1 & 1 & 1 \\
$\boldsymbol{C s}_{\mathbf{3}}(\boldsymbol{E}, \boldsymbol{F})$ & 0.71 & 1 & 1 & 1 \\
\hline
\end{tabular}

Table 1. Values of similarity measures by using Eqs.(1-4), and (7-9) 
Also, the balanced values of similarity by the three types of proposed measures $C s_{1}(E, F)$ ,$C s_{2}(E, F)$ and $C s_{3}(E, F)$ produces a reasonable phenomenon for the similarity measures between $E$ and $F$. So, they are appropriate to handle medical diagnosis problems.

\section{Proposed decision making algorithms}

Based on proposed measures of similarity, we present in this section two MADM techniques under BNSs and IVBNSs environments.

\subsection{MADM algorithm for bipolar neutrosophic sets (BNSs)}

The method for solving MADM problems under BNS environment is introduced as follows:

Step 1. Construct decision matrix

Let $A_{1}, A_{2}, \ldots, A_{m}$ be a group of alternatives and $C_{1}, C_{2}, \ldots, C_{n}$ are the attributes. The decision maker begin to construct a decision matrix $D_{m \times n}=$ $\left\langle\Gamma^{+}{ }_{i j}, \xi^{+}{ }_{i j}, \Psi^{+}{ }_{i j}, \Gamma^{-}{ }_{i j}, \xi^{-}{ }_{i j}, \Psi^{-}{ }_{i j}\right\rangle_{m \times n}$ with respect to $m$ alternatives and $n$ attributes in terms of bipolar neutrosophic sets , for $i=1,2, \ldots, m$ and $j=1,2, \ldots, n$. The bipolar neutrosophic decision matrix as in Table 2 as follows:

\begin{tabular}{|c|c|c|}
\hline$C_{1}$ & $C_{2}$ & $C_{n}$ \\
\hline $\begin{array}{ll}A_{1} & \left\langle\Gamma^{+}{ }_{11}, \xi^{+}{ }_{11}, \Psi^{+}{ }_{11}, \Gamma^{-}{ }_{11}, \xi^{-}{ }_{11}, \Psi^{-}{ }_{11}\right\rangle\end{array}$ & $\left\langle\Gamma^{+}{ }_{12}, \xi^{+}{ }_{12}, \Psi^{+}{ }_{12}, \Gamma^{-}{ }_{12}, \xi^{-}{ }_{12}, \Psi^{-}{ }_{12}\right\rangle$ & $\ldots \quad\left\langle\Gamma^{+}{ }_{1 n}, \xi^{+}{ }_{1 n}, \Psi^{+}{ }_{1 n}, \Gamma^{-}{ }_{1 n}, \xi^{-}{ }_{1 n}, \Psi^{-}{ }_{1 n}\right\rangle$ \\
\hline $\boldsymbol{A}_{2}\left\langle\left\langle\Gamma^{+}{ }_{21}, \xi^{+}{ }_{21}, \Psi^{+}{ }_{21}, \Gamma^{-}{ }_{21}, \xi^{-}{ }_{21}, \Psi^{-}{ }_{21}\right\rangle\right.$ & $\left\langle\Gamma^{+}{ }_{22}, \xi^{+}{ }_{22}, \Psi^{+}{ }_{22}, \Gamma^{-}{ }_{22}, \xi^{-}{ }_{22}, \Psi^{-}{ }_{22}\right\rangle$ & $\ldots \quad\left\langle\Gamma^{+}{ }_{2 n}, \xi^{+}{ }_{2 n}, \Psi^{+}{ }_{2 n}, \Gamma^{-}{ }_{2 n}, \xi^{-}{ }_{2 n}, \Psi^{-}{ }_{2 n}\right\rangle$ \\
\hline$\ldots$ & $\ldots$ & $\ldots$ \\
\hline . & $\ldots$ & $\ldots$ \\
\hline$\xi^{+}{ }_{m 1}, \Psi^{+}{ }_{m 1}, \Gamma_{m 1}^{-}, \xi_{m 1}^{-}, \Psi^{-}$ & ${ }_{m 2}, \xi^{+}{ }_{m 2}, \Psi^{+}{ }_{m 2}, \Gamma_{m 2}^{-}, \xi^{-}{ }_{m 2}, \Psi_{m}^{-}$ & $\ldots \quad\left\langle\Gamma^{+}{ }_{m n}, \xi^{+}{ }_{m n}, \Psi^{+}{ }_{m n}, \Gamma^{-}{ }_{m n}, \xi^{-}{ }_{m n^{\prime}}, \Psi^{-}{ }_{r}\right.$ \\
\hline
\end{tabular}

Table 2. Bipolar neutrosophic decision matrix

Step 2. Determine weights for attributes.

Let decision maker determine weights $w_{j}(j=1,2, \ldots, n)$ of attributes $C_{j}(j=1,2, \ldots, n)$, since $w_{j} \in[0,1]$ and $\sum_{j=1}^{n} w_{j}=1$.

Step 3. Determination of optimal sequence of the attribute values according to problem domain.

According to problem domain, let decision maker begin to construct the ideal sequence of attributes values, $I d^{*}=\left\{\left\langle\Gamma^{+^{*}}{ }_{j}, \xi^{+}{ }_{j}{ }^{*}, \Psi^{+_{j}}{ }^{*}, \Gamma^{-}{ }_{j}{ }^{*}, \xi^{-}{ }_{j}{ }^{*}, \Psi^{-}{ }_{j}{ }^{*}\right\rangle, \ldots, \ldots\right\}, j=1,2, \ldots, n$.

Step 4. Calculation of similarity measures.

Develop weighted cosine similarity measures for BNSs to measure similarity among $A_{i}$ and $I d^{*}$ as follows:

$$
\begin{aligned}
& C s_{1}{ }^{w}\left(A_{i}, I d^{*}\right)=\sum_{j=1}^{n} w_{j} \times \cos \left(\frac{\pi}{12} \times\left(\left|\Gamma^{+}{ }_{i j}-\Gamma^{+^{*}}{ }_{j}\right|+\left|\xi^{+}{ }_{i j}-\xi^{+}{ }_{j}\right|+\mid \Psi^{+}{ }_{i j}-\right.\right. \\
& \left.\left.\Psi^{+{ }_{j}}{ }^{*}|+| \Gamma^{-}{ }_{i j}-\Gamma^{-{ }_{j}}|+| \xi^{-}{ }_{i j}-\xi^{-{ }_{j}}|+| \Psi^{-}{ }_{i j}-\Psi^{-}{ }_{j}{ }^{*} \mid\right)\right)
\end{aligned}
$$




$$
\begin{aligned}
& C s_{2}{ }^{w}\left(A_{i}, I d^{*}\right)=\sum_{j=1}^{n} w_{j} \times \cos \left(\frac{\pi}{4} \times \operatorname{Max}\left(\left|\Gamma^{+}{ }_{i j}-\Gamma^{+{ }^{*}}\right|,\left|\xi^{+}{ }_{i j}-\xi^{+}{ }_{j}{ }^{*}\right|, \mid \Psi^{+}{ }_{i j}-\right.\right. \\
& \left.\left.\Psi^{+{ }_{j}^{*}}|,| \Gamma^{-}{ }_{i j}-\Gamma^{-{ }_{j}}|,| \xi^{-}{ }_{i j}-\xi^{-}{ }_{j}|,| \Psi^{-}{ }_{i j}-\Psi_{j}^{-}{ }_{j} \mid\right)\right) \\
& \operatorname{Cs}_{3}{ }^{w}\left(A_{i}, I d^{*}\right)=\sum_{j=1}^{n} w_{j} \times \cos \left(\frac{\pi}{2} \times\left(\frac{M_{1}+M_{2}}{2}\right)\right) \\
& \text { Since, } \quad w_{j} \in[0,1], M_{1}=\operatorname{Max}\left(\left|\Gamma^{+}{ }_{i j}-\Gamma^{+^{*}}{ }_{j}\right|,\left|\xi^{+}{ }_{i j}-\xi^{+}{ }_{j}{ }^{*}\right|,\left|\Psi^{+}{ }_{i j}-\Psi^{+}{ }_{j}{ }^{*}\right|, \mid \Gamma^{-}{ }_{i j}-\right. \\
& \left.\Gamma^{-}{ }_{j}^{*}|,| \xi^{-}{ }_{i j}-\xi^{-}{ }_{j}^{*}|,| \Psi^{-}{ }_{i j}-\Psi^{-}{ }_{j}{ }^{*} \mid\right) \text {, } \\
& M_{2}=\operatorname{Min}\left(\left|\Gamma^{+}{ }_{i j}-\Gamma^{+^{*}}{ }_{j}\right|,\left|\xi^{+}{ }_{i j}-\xi^{+}{ }_{j}{ }^{*}\right|,\left|\Psi^{+}{ }_{i j}-\Psi^{+}{ }_{j}{ }^{*}\right|,\left|\Gamma^{-}{ }_{i j}-\Gamma^{-}{ }_{j}{ }^{*}\right|, \mid \xi^{-}{ }_{i j}-\right. \\
& \left.\xi^{-*}|,| \Psi^{-}{ }_{i j}-\Psi^{-}{ }_{j}^{*} \mid\right) \text {. }
\end{aligned}
$$

Step 5. Rank alternatives.

The alternative with the biggest similarity measure is the best one.

\subsection{MADM algorithm for interval valued bipolar neutrosophic set (IVBNS) environment}

The method to solve MADM problems under IVBNS environment is introduced as follows:

Step 1. Construct decision matrix.

Let $A_{1}, A_{2}, \ldots, A_{m}$ be a group of alternatives and $C_{1}, C_{2}, \ldots, C_{n}$ are the attributes. The decision maker begin to construct a decision matrix $D_{m \times n}=$ $\left\langle\left[\Gamma_{i j}^{+L}, \Gamma_{i j}^{+U}\right],\left[\xi_{i j}^{+L}, \xi_{i j}^{+U}\right],\left[\Psi_{i j}^{+L}, \Psi_{i j}^{+U}\right],\left[\Gamma_{i j}^{-L}, \Gamma_{i j}^{-U}\right],\left[\xi_{i j}^{-L}, \xi_{i j}^{-U}\right],\left[\Psi_{i j}^{-L}, \Psi_{i j}^{-U}\right]\right\rangle_{m \times n}$ with respect to $m$ alternatives and $n$ attributes in terms of interval valued bipolar neutrosophic sets , for $i=$ $1,2, \ldots, m$ and $j=1,2, \ldots, n$.

Step 2. Determine weights for attributes.

Let decision maker determine weights $w_{j}(j=1,2, \ldots, n)$ of attributes $C_{j}(j=1,2, \ldots, n)$, since $w_{j} \in[0,1]$ and $\sum_{j=1}^{n} w_{j}=1$.

Step 3. Determination of optimal sequence of the attribute values according to problem domain.

According to problem domain, let decision maker begin to construct the ideal sequence of attributes values, $\quad \mathrm{Id}^{*}=$ $\left\{\left\langle\left[\Gamma_{j}^{+L^{*}}, \Gamma_{j}^{+U *}\right],\left[\xi_{j}^{+{ }^{L *}}, \xi_{j}^{+U *}\right],\left[\Psi_{j}^{+L^{*}}, \Psi_{j}^{+U *}\right],\left[\Gamma_{j}^{-L *}, \Gamma_{j}^{-U *}\right],\left[\xi_{j}^{-L *}, \xi_{j}^{-U *}\right],\left[\Psi_{j}^{-L *}, \Psi_{j}^{-U *}\right]\right\rangle, \ldots, \ldots\right\}, j=$

$1,2, \ldots, n$.

Step 4. Calculation of similarity measures.

Develop weighted cosine similarity measures for interval valued bipolar neutrosophic sets to measure similarity among $A_{i}$, and $I d^{*}$ as follows: 


$$
\begin{aligned}
& C s_{1}{ }^{w}\left(A_{i}, I d^{*}\right)=\sum_{j=1}^{n} w_{j} \times \cos \left(\frac{\pi}{24} \times\left(\left|\Gamma_{i j}^{+{ }^{L}}-\Gamma_{j}^{+{ }^{L *}}\right|+\left|\Gamma_{i j}^{+{ }^{U}}-\Gamma_{j}^{+{ }^{U *}}\right|+\mid \xi_{i j}^{{ }^{L}}-\right.\right. \\
& \xi_{j}^{+L *}|+| \xi_{i j}^{+U}-\xi_{j}^{+U *}|+| \Psi_{i j}^{+{ }^{L}}-\Psi_{j}^{+L *}|+| \Psi_{i j}^{+U}-\Psi_{j}^{+U *}|+| \Gamma_{i j}^{-L}-\Gamma_{j}^{-L *}|+| \Gamma_{i j}^{-U}-\Gamma_{j}^{-U *} \mid+ \\
& \left.\left.\left|\xi_{i j}^{-L}-\xi_{j}^{-L *}\right|+\left|\xi_{i j}^{-U}-\xi_{j}^{-U *}\right|+\left|\Psi_{i j}^{-L}-\Psi_{j}^{-L *}\right|+\left|\Psi_{i j}^{-U}-\Psi_{j}^{-U *}\right|\right)\right) \\
& C s_{2}{ }^{w}\left(A_{i}, I d^{*}\right)=\sum_{j=1}^{n} w_{j} \times \cos \left(\frac{\pi}{4} \times \operatorname{Max}\left(\left|\Gamma_{i j}^{+{ }^{L}}-\Gamma_{j}^{+{ }^{L *}}\right|,\left|\Gamma_{i j}^{+{ }^{U}}-\Gamma_{j}^{+U *}\right|, \mid \xi_{i j}^{+{ }^{L}}-\right.\right. \\
& \xi_{j}^{+L^{*}}|,| \xi_{i j}^{+U}-\xi_{j}^{+U *}|,| \Psi_{i j}^{+{ }^{L}}-\Psi_{j}^{+L^{*}}|,| \Psi_{i j}^{+U}-\Psi_{j}^{+{ }^{U *}}|,| \Gamma_{i j}^{-L^{L}}-\Gamma_{j}^{-L^{*}}|,| \Gamma_{i j}^{-U}-\Gamma_{j}^{-U *}|,| \xi_{i j}^{-L^{L}}- \\
& \left.\left.\xi_{j}^{-L *}|,| \xi_{i j}^{-U}-\xi_{j}^{-U *}|,| \Psi_{i j}^{-L}-\Psi_{j}^{-L *}|,| \Psi_{i j}^{-U}-\Psi_{j}^{-U *} \mid\right)\right) \\
& \operatorname{Cs}_{3}{ }^{w}\left(A_{i}, I d^{*}\right)=\sum_{j=1}^{n} w_{j} \times \cos \left(\frac{\pi}{2} \times\left(\frac{M_{1}+M_{2}}{2}\right)\right)
\end{aligned}
$$

Since,

$$
\begin{aligned}
& w_{j} \in[0,1], M_{1}=\operatorname{Max}\left(\left|\Gamma_{i j}^{+{ }^{L}}-\Gamma_{j}^{+{ }^{*}}\right|,\left|\Gamma_{i j}^{+U}-\Gamma_{j}^{+U *}\right|,\left|\xi_{i j}^{+{ }^{L}}-\xi_{j}^{L^{L *}}\right|,\left|\xi_{i j}^{+U^{U}}-\xi_{j}^{+U^{*}}\right|, \mid \Psi_{i j}^{+{ }^{L}}-\right. \\
& \Psi_{j}^{+L *}|,| \Psi_{i j}^{+U}-\Psi_{j}^{+U *}|,| \Gamma_{i j}^{-L}-\Gamma_{j}^{-L *}|,| \Gamma_{i j}^{-U}-\Gamma_{j}^{-U *}|,| \xi_{i j}^{-L}-\xi_{j}^{-L *}|,| \xi_{i j}^{-U}-\xi_{j}^{-U *}|,| \Psi_{i j}^{-L}- \\
& \left.\Psi_{j}^{-L *}|,| \Psi_{i j}^{-U}-\Psi_{j}^{-U *} \mid\right) \text {, } \\
& M_{2}=\operatorname{Min}\left(\left|\Gamma_{i j}^{+{ }^{L}}-\Gamma_{j}^{+L^{*}}\right|,\left|\Gamma_{i j}^{+U}-\Gamma_{j}^{+U *}\right|,\left|\xi_{i j}^{+{ }^{L}}-\xi_{j}^{+L^{*}}\right|,\left|\xi_{i j}^{+U}-\xi_{j}^{+U^{*}}\right|,\left|\Psi_{i j}^{+{ }^{L}}-\Psi_{j}^{+L^{*}}\right|, \mid \Psi_{i j}^{+U}-\right. \\
& \left.\Psi_{j}^{+U *}|,| \Gamma_{i j}^{-L}-\Gamma_{j}^{-L *}|,| \Gamma_{i j}^{-U}-\Gamma_{j}^{-U *}|,| \xi_{i j}^{-L}-\xi_{j}^{-L *}|,| \xi_{i j}^{-U}-\xi_{j}^{-U *}|,| \Psi_{i j}^{-L}-\Psi_{j}^{-L *}|,| \Psi_{i j}^{-U}-\Psi_{j}^{-U *} \mid\right) \text {. }
\end{aligned}
$$

Step 5. Rank alternatives.

The alternative with the biggest similarity measure is the best one.

\section{Numerical examples}

We solved in this section two numerical multi-attribute decision making problems and compared outputs with other methods for verifying the applicability and effectiveness of the suggested methods under BNS and IVBNS environments.

\subsection{Numerical example 1}

We present the multi-attribute decision making problem studied by Ulucay et al. [43] for BNS in this example. The car company needs to select the best green supplier from available suppliers. The company's manager taken into his consideration four alternatives (suppliers) which denoted by $A_{1}, A_{2}, A_{3}$, and $A_{4}$. Also, three attributes are considered which are product quality $\left(C_{1}\right)$, technology capability $\left(C_{2}\right)$, and pollution control $\left(C_{3}\right)$. The three criteria's weights are $0.2,0.5,0.3$ respectively. The decision maker has determined the values of attributes for the four suppliers (alternatives) under BNS environment to determine decision information. These assessment values are presented in the decision matrix $D_{m \times n}=\left\langle\Gamma^{+}{ }_{i j}, \xi^{+}{ }_{i j}, \Psi^{+}{ }_{i j}, \Gamma^{-}{ }_{i j}, \xi^{-}{ }_{i j}, \Psi^{-}{ }_{i j}\right\rangle_{4 \times 3}$, since $i=1,2,3,4$ and $j=1,2,3$. The proposed steps for solving this problem are as follows:

Step 1. Build problem's decision matrix.

The decision matrix of four alternatives according to three attributes in terms of BNS is presented in Table 3. 


\begin{tabular}{cccc}
\hline & $\boldsymbol{C}_{\mathbf{1}}$ & $\boldsymbol{C}_{\mathbf{2}}$ & $\boldsymbol{C}_{3}$ \\
\hline $\boldsymbol{A}_{\mathbf{1}}$ & $\langle 0.4,0.5,0.3,-0.6,-0.4,-0.5\rangle$ & $\langle 0.6,0.1,0.2,-0.4,-0.3,-0.2\rangle$ & $\langle 0.8,0.6,0.5,-0.3,-0.2,-0.1\rangle$ \\
$\boldsymbol{A}_{\mathbf{2}}$ & $\langle 0.6,0.4,0.2,-0.4,-0.5,-0.7\rangle$ & $\langle 0.6,0.2,0.3,-0.5,-0.2,-0.3\rangle$ & $\langle 0.7,0.4,0.5,-0.1,-0.3,-0.4\rangle$ \\
$\boldsymbol{A}_{3}$ & $\langle 0.7,0.2,0.4,-0.2,-0.6,-0.4\rangle$ & $\langle 0.9,0.3,0.6,-0.2,-0.2,-0.5\rangle$ & $\langle 0.6,0.1,0.5,-0.2,-0.4,-0.6\rangle$ \\
$\boldsymbol{A}_{\mathbf{4}}$ & $\langle 0.8,0.6,0.5,-0.5,-0.3,-0.6\rangle$ & $\langle 0.6,0.4,0.3,-0.1,-0.3,-0.4\rangle$ & $\langle 0.9,0.6,0.4,-0.5,-0.3,-0.6\rangle$ \\
\hline
\end{tabular}

Table 3. Bipolar neutrosophic decision matrix

Step 2. Decide weights of attributes.

The decision maker determined weights of attributes as $0.2,0.5$ and 0.3 respectively.

Step 3. Determine optimal sequence of the attribute values according to problem domain. The decision maker constructed the ideal sequence of attributes values as follows: $I d^{*}=$ $\{\langle 1,0,0,0,-1,-1\rangle,\langle 1,0,0,0,-1,-1\rangle,\langle 1,0,0,0,-1,-1\rangle\}$.The reason for selecting this ideal sequence of attributes values returned to decision maker's opinion. Since we usually want to obtain solutions with the highest truth degree, and lowest indeterminacy and falsity degrees, then the maximum value of positive and negative truth membership should be selected and also the minimum value of positive and negative indeterminacy and falsity degrees should be selected. Thus, the optimal value of positive truth membership degree for bipolar neutrosophic set is 1 , and for negative truth membership degree is 0 . Also, the optimal value of positive indeterminacy and falsity membership degrees for bipolar neutrosophic set is 0 , and for negative indeterminacy and falsity membership degrees is -1 . Based on this concept the decision maker selected the optimal sequence of attributes values in this problem.

Step 4. Calculate similarity measures.

Use Eqs. (19-21) for calculating similarity measures between alternatives and optimal sequence of attribute values $I d^{*}$.

Step 5. Rank alternatives.

The ranking and similarity measures results presented in Table 4.

\begin{tabular}{|c|c|c|}
\hline Similarity measures & Measure values & Ranking order \\
\hline$C s_{1}{ }^{w}\left(A_{1}, I d^{*}\right)$ & 0.7211 & \multirow{5}{*}{$A_{3}>A_{4}>A_{2}>A_{1}$} \\
\hline$C s_{1}{ }^{w}\left(A_{2}, I d^{*}\right)$ & 0.7636 & \\
\hline$C s_{1}{ }^{w}\left(A_{3}, I d^{*}\right)$ & 0.8188 & \\
\hline$C s_{1}{ }^{w}\left(A_{4}, I d^{*}\right)$ & 0.7698 & \\
\hline$C s_{2}{ }^{w}\left(A_{1}, I d^{*}\right)$ & 0.8108 & \\
\hline$C s_{2}{ }^{w}\left(A_{2}, I d^{*}\right)$ & 0.8308 & \multirow{3}{*}{$A_{4}>A_{3}>A_{2}>A_{1}$} \\
\hline$C s_{2}{ }^{w}\left(A_{3}, I d^{*}\right)$ & 0.8500 & \\
\hline$C s_{2}{ }^{w}\left(A_{4}, I d^{*}\right)$ & 0.8526 & \\
\hline$C s_{3}{ }^{w}\left(A_{1}, I d^{*}\right)$ & 0.7271 & \multirow{4}{*}{$A_{4}>A_{3}>A_{2}>A_{1}$} \\
\hline$C s_{3}{ }^{w}\left(A_{2}, I d^{*}\right)$ & 0.7483 & \\
\hline$C s_{3}{ }^{w}\left(A_{3}, I d^{*}\right)$ & 0.7977 & \\
\hline$C s_{3}{ }^{w}\left(A_{4}, I d^{*}\right)$ & 0.7992 & \\
\hline
\end{tabular}

Table 4. Similarity measures values and ranking of alternatives

\subsubsection{Results and comparison}

For showing superiority and applicability of the proposed method, we present these comparisons. Then, various methods are used for solving the same problem. The ranking order of alternatives by various methods presented in Table 5. As appears in Table 5 there exist some differences among ranking results of alternatives by the presented methods. The 
three proposed types of cosine similarity measures reflect that $A_{1}$ is the worst alternative. Also, in proposed methods by Deli et al. [41] and Sahin et al. [42], the first alternative $A_{1}$ is also the worst alternative, and this consist with the three types of proposed measures. But, $A_{1}$ is the best alternative according to Ulucay et al. [43] when $\lambda=0.9$. The second and third type of proposed cosine similarity measure have the same results of ranking order, and there is a slight difference with the first proposed type. Also, as appears in Table 5, the ranking process by the first type of proposed cosine similarity measure is exactly as obtained by Sahin et al. [42] method. The best alternatives according to Ulucay et al. [43] when $\lambda=0.3$ and 0.6 is $A_{3}$. It also the best alternative according to Sahin et al. [42], Deli and Subas's method [46], and our proposed method of first type of cosine similarity measure. Although results of ranking order for alternatives by the first proposed measure of similarity are exactly as in [42], and similar in optimal alternative with $[43,46]$, our proposed measures are very simple, with the minimum computational burden as compared with others $[41,42,43,46]$, and cannot produce unreasonable results. Therefore, the proposed measures are superior to the existing measures and cannot produce any conflict of some cases as in $[42,43]$, as we have shown in section 3 .

\begin{tabular}{cc}
\hline Methods & Ranking order \\
\hline $\begin{array}{c}\text { First type of proposed cosine } \\
\text { similarity measure }\end{array}$ & $A_{3}>A_{4}>A_{2}>A_{1}$ \\
\hline Deli et al.[41] & $A_{2}>A_{3}>A_{4}>A_{1}$ \\
\hline $\begin{array}{c}\text { Second type of proposed } \\
\text { cosine similarity measure }\end{array}$ & $A_{4}>A_{3}>A_{2}>A_{1}$ \\
\hline $\begin{array}{c}\text { Third type of proposed cosine } \\
\text { similarity measure }\end{array}$ & $A_{4}>A_{3}>A_{2}>A_{1}$ \\
\hline Sahin et al.[42] & $A_{3}>A_{4}>A_{2}>A_{1}$ \\
\hline $\begin{array}{c}\text { Ulucay et al.[43] when } \boldsymbol{\lambda}=\mathbf{0 . 3} \\
\text { Ulucay et al.[43] when } \boldsymbol{\lambda}=\mathbf{0 . 6}\end{array}$ & $A_{3}>A_{1}>A_{4}>A_{2}$ \\
\hline Ulucay et al.[43] when $\boldsymbol{\lambda}=\mathbf{0 . 9}$ & $A_{1}>A_{3}>A_{4}>A_{2}$ \\
\hline Deli and Subas's method [46] & $A_{3}>A_{1}>A_{4}>A_{2}$ \\
\hline
\end{tabular}

Table 5. Ranking order of alternatives by existing methods

\subsection{Numerical example 2}

For illustrating proposed algorithm of interval valued bipolar neutrosophic information for decision making problems, we consider the following example:

We study the MADM problem studied by Pramanik et al. [47] with four available alternatives for investing a sum of money and based on three attributes. The possible alternatives are as follows:

a) Food company $\left(A_{1}\right)$, 
b) Car company $\left(A_{2}\right)$,

c) Arm company $\left(A_{3}\right)$,

d) Car computer $\left(A_{4}\right)$.

The three determined attributes are as follows:

a) Growth analysis $\left(C_{1}\right)$,

b) Risk analysis $\left(C_{2}\right)$, and

c) Environment analysis $\left(C_{3}\right)$.

The weight of attributes is $0.35,0.25$, and 0.40 respectively. Perform the following steps for solving this problem:

Step 1. Build problem's decision matrix.

The decision matrix of four alternatives according to three attributes in terms of interval valued bipolar neutrosophic sets is presented in Table 6 .

\begin{tabular}{cc}
\hline & $\boldsymbol{C}_{1}$ \\
\hline $\boldsymbol{A}_{\mathbf{1}}$ & $\langle[0.4,0.5],[0.2,0.3],[0.3,0.4],[-0.3,-0.2],[-0.4,-0.3],[-0.5,-0.4]\rangle$ \\
$\boldsymbol{A}_{\mathbf{2}}$ & $\langle[0.6,0.7],[0.1,0.2],[0.2,0.3],[-0.2,-0.1],[-0.3,-0.2],[-0.7,-0.6]\rangle$ \\
$\boldsymbol{A}_{3}$ & $\langle[0.3,0.6],[0.2,0.3],[0.3,0.4],[-0.3,-0.2],[-0.4,-0.3],[-0.6,-0.3]\rangle$ \\
$\boldsymbol{A}_{\mathbf{4}}$ & $\langle[0.7,0.8],[0.0,0.1],[0.1,0.2],[-0.1,-0.0],[-0.2,-0.1],[-0.8,-0.7]\rangle$ \\
& $\boldsymbol{C}_{2}$ \\
$\boldsymbol{A}_{\mathbf{1}}$ & $\langle[0.4,0.6],[0.1,0.3],[0.2,0.4],[-0.3,-0.1],[-0.4,-0.2],[-0.6,-0.4]\rangle$ \\
$\boldsymbol{A}_{\mathbf{2}}$ & $\langle[0.6,0.7],[0.1,0.2],[0.2,0.3],[-0.2,-0.1],[-0.3,-0.2],[-0.7,-0.6]\rangle$ \\
$\boldsymbol{A}_{3}$ & $\langle[0.5,0.6],[0.2,0.3],[0.3,0.4],[-0.3,-0.2],[-0.4,-0.3],[-0.6,-0.5]\rangle$ \\
$\boldsymbol{A}_{4}$ & $\langle[0.6,0.7],[0.1,0.2],[0.1,0.3],[-0.2,-0.1],[-0.3,-0.1],[-0.7,-0.6]\rangle$ \\
& $\boldsymbol{C}_{3}$ \\
$\boldsymbol{A}_{\mathbf{1}}$ & $\langle[0.7,0.9],[0.2,0.3],[0.4,0.5],[-0.3,-0.2],[-0.5,-0.4],[-0.9,-0.7]\rangle$ \\
$\boldsymbol{A}_{\mathbf{2}}$ & $\langle[0.3,0.6],[0.3,0.5],[0.8,0.9],[-0.5,-0.3],[-0.9,-0.8],[-0.6,-0.3]\rangle$ \\
$\boldsymbol{A}_{3}$ & $\langle[0.4,0.5],[0.2,0.4],[0.7,0.9],[-0.4,-0.2],[-0.9,-0.7],[-0.5,-0.4]\rangle$ \\
$\boldsymbol{A}_{4}$ & $\langle[0.6,0.7],[0.3,0.4],[0.8,0.9],[-0.4,-0.3],[-0.9,-0.8],[-0.7,-0.6]\rangle$ \\
\hline
\end{tabular}

Table 6. Interval valued bipolar neutrosophic decision matrix

Step 2. Determine attributes weights.

Decision makers determined weights of attributes as $0.35,0.25$, and 0.40 respectively.

Step 3. Determine optimal sequence of the attributes values according to problem domain Decision makers constructed the ideal sequence of attributes values as follows: $\mathrm{Id}^{*}=$ $\langle[1,1],[0,0],[0,0],[0,0],[-1,-1],[-1,-1]\rangle,\langle[1,1],[0,0],[0,0],[0,0],[-1,-1],[-1,-1]\rangle$ $\langle[1,1],[0,0],[0,0],[0,0],[-1,-1],[-1,-1]\rangle$.

Since it's the optimal sequence of interval valued bipolar neutrosophic sets because we usually want to maximize truthiness and minimize falsity and indeterminacy for obtaining solutions.

Step 4. Calculate similarity measures.

Use Eqs. (22-24) for calculating similarity measures between alternatives and ideal sequence of attribute values $I d^{*}$.

Step 5. Rank alternatives.

The ranking and similarity measures results presented in Table 7 . 


\begin{tabular}{|c|c|c|}
\hline Similarity measures & Measure values & Ranking order \\
\hline$C s_{1}{ }^{w}\left(A_{1}, I d^{*}\right)$ & 0.8257 & \multirow{5}{*}{$A_{4}>A_{1}>A_{2}>A_{3}$} \\
\hline$C s_{1}{ }^{w}\left(A_{2}, I d^{*}\right)$ & 0.8097 & \\
\hline$C s_{1}{ }^{w}\left(A_{3}, I d^{*}\right)$ & 0.7784 & \\
\hline$C s_{1}{ }^{w}\left(A_{4}, I d^{*}\right)$ & 0.8598 & \\
\hline$C s_{2}{ }^{w}\left(A_{1}, I d^{*}\right)$ & 0.8571 & \\
\hline$C s_{2}{ }^{w}\left(A_{2}, I d^{*}\right)$ & 0.7895 & \multirow{4}{*}{$A_{1}>A_{3}>A_{2}>A_{4}$} \\
\hline$C s_{2}{ }^{w}\left(A_{3}, I d^{*}\right)$ & 0.8157 & \\
\hline$C s_{2}{ }^{w}\left(A_{4}, I d^{*}\right)$ & 0.7604 & \\
\hline$C s_{3}{ }^{w}\left(A_{1}, I d^{*}\right)$ & 0.7972 & \\
\hline$C s_{3}{ }^{w}\left(A_{2}, I d^{*}\right)$ & 0.7390 & \multirow[t]{3}{*}{$A_{1}>A_{3}>A_{2}>A_{4}$} \\
\hline$C s_{3}{ }^{w}\left(A_{3}, I d^{*}\right)$ & 0.7390 & \\
\hline$C s_{3}{ }^{w}\left(A_{4}, I d^{*}\right)$ & 0.7257 & \\
\hline
\end{tabular}

Table 7. Similarity measures values and ranking order of alternatives

\subsubsection{Results and comparison}

The ranking order of alternatives by various methods presented in Table 8. As appears in Table 8 there exist some differences among the results of ranking process which obtained by the presented methods. The first type of proposed cosine similarity measure and proposed methods in $[48,49]$ agreed that $A_{4}$ is the best choice. But, second and third type of proposed cosine similarity measures agreed that $A_{1}$ is the best choice. The ranking result obtained from [47] differs from the optimal result of other methods.

\begin{tabular}{ccc}
\hline Methods & Ranking order & Best choice \\
\hline $\begin{array}{c}\text { First type of proposed cosine } \\
\text { similarity measure }\end{array}$ & $A_{4}>A_{1}>A_{2}>A_{3}$ & $A_{4}$ \\
$\begin{array}{c}\text { Second type of proposed } \\
\text { cosine similarity measure }\end{array}$ & $A_{1}>A_{3}>A_{2}>A_{4}$ & $A_{1}$ \\
$\begin{array}{c}\text { Third type of proposed cosine } \\
\text { similarity measure }\end{array}$ & $A_{1}>A_{3}>A_{2}>A_{4}$ & $A_{1}$ \\
Proposed method in [47] & $A_{2}>A_{4}>A_{3}>A_{1}$ & $A_{2}$ \\
Proposed method in [48] & $A_{4}>A_{1}>A_{3}>A_{2}$ & $A_{4}$ \\
\hline Proposed method in [49] & $A_{4}>A_{2}>A_{3}>A_{1}$ & $A_{4}$ \\
\hline
\end{tabular}

Table 8. Ranking order of alternatives by existing methods

\section{Applying proposed measures of similarity in medical diagnosis of bipolar disorder disease}

The proposed methods of similarity are applied in this section for diagnosing bipolar disorder disease as shown below:

Bipolar disorder causes obvious changes in energy, mood, and activity levels. These changes range from manic episodes (which means periods of exceedingly "up," elated, and 
active behavior), to depressive periods (which means periods of very sad, "down," or hopeless periods). Mood episodes of people with bipolar disorder are radically various from the moods which are usual for the person.

About $3 \%$ of the total population over the age of 18 in the U.S suffer from bipolar disorder according to estimates of National Institute of Mental Health, but only 51\% of people with bipolar disorder receive treatment. People who left untreated from bipolar disorder will often have an increased rate in riskiness and may lead to suicide, since there is a high suicide rate for people with the disorder. Therefore, we must diagnose the disease and determine the method of treatment for controlling the symptoms and enjoying a more stable life and satisfaction.

\subsection{Case study}

In this sub-section we consider a medical diagnosis problem to illustrate the applicability of the proposed measures of similarity.

Let $P e=\left\{P e_{1}, P e_{2}, P e_{3}, P e_{4}\right\}$ be a group of persons with various states of mood and behaviors as follows: $S_{1}$ (happiness), $S_{2}$ (sadness), $S_{3}$ (energy), $S_{4}$ (sleeping rate). Here we want to examine people to determine if one or more person suffer from bipolar disorder or not, and then identify patients for treatment. The steps for making this diagnosis are as follows:

Step 1. Build decision matrix of problem.

For constructing decision matrix, we spoke with a mental health professional (decision maker). The doctor made a physical exam to ensure that these symptoms did not caused by other illnesses, and after making a mental health evaluation of persons (alternatives) the decision matrix of four alternatives with respect to four states (attributes) presented in Table 9, in terms of interval valued bipolar neutrosophic sets. The reason for representing decision matrix under interval valued bipolar neutrosophic environment returned to the nature of disease, since unusual moves in energy, mood, and the capability to do daily tasks ranges from "down" and "up".

\begin{tabular}{|c|c|}
\hline & $S_{1}$ \\
\hline$P e_{1}$ & $\langle[0.3,0.4],[0.2,0.9],[0.3,0.8],[-0.8,-0.0],[-0.9,-0.0],[-0.8,-0.6]\rangle$ \\
\hline $\mathrm{Pe}_{2}$ & $\langle[1.0,1.0],[0.9,1.0],[1.0,1.0],[-0.9,-0.8],[-1.0,-0.9],[-0.9,-0.8]\rangle$ \\
\hline$P e_{3}$ & $\langle[0.3,0.6],[0.2,0.3],[0.3,0.4],[-0.3,-0.2],[-0.4,-0.3],[-0.6,-0.3]\rangle$ \\
\hline $\mathrm{Pe}_{4}$ & $\langle[0.9,1.0],[0.9,1.0],[0.8,0.9],[-1.0,-0.0],[-0.9,-0.8],[-0.8,-0.7]\rangle$ \\
\hline & $S_{2}$ \\
\hline$P e_{1}$ & $\langle[0.0,0.8],[0.1,0.9],[0.2,1.0],[-1.0,-0.0],[-1.0,-0.0],[-0.8,-0.0]\rangle$ \\
\hline $\mathrm{Pe}_{2}$ & $\langle[1.0,1.0],[0.9,1.0],[1.0,1.0],[-0.9,-0.8],[-1.0,-0.9],[-0.9,-0.8]\rangle$ \\
\hline $\mathrm{Pe}_{3}$ & $\langle[0.5,0.6],[0.3,0.5],[0.4,0.6],[-0.7,-0.4],[-0.8,-0.4],[-0.7,-0.5]\rangle$ \\
\hline $\mathrm{Pe}_{4}$ & $\langle[0.9,1.0],[0.9,1.0],[0.8,0.9],[-1.0,-0.0],[-0.9,-0.8],[-0.8,-0.7]\rangle$ \\
\hline & $S_{3}$ \\
\hline$P e_{1}$ & $\langle[0.3,0.4],[0.2,0.9],[0.3,0.8],[-0.8,-0.0],[-0.9,-0.0],[-0.8,-0.7]\rangle$ \\
\hline $\mathrm{Pe}_{2}$ & $\langle[1.0,1.0],[0.9,1.0],[1.0,1.0],[-0.9,-0.8],[-1.0,-0.9],[-0.9,-0.8]\rangle$ \\
\hline $\mathrm{Pe}_{3}$ & $\langle[0.4,0.5],[0.2,0.4],[0.7,0.9],[-0.4,-0.2],[-0.9,-0.7],[-0.5,-0.4]\rangle$ \\
\hline $\mathrm{Pe}_{4}$ & $\langle[0.8,0.9],[0.7,0.9],[0.8,0.9],[-0.9,-0.0],[-0.8,-0.7],[-0.8,-0.7]\rangle$ \\
\hline & \\
\hline
\end{tabular}




\begin{tabular}{ll}
\hline$P e_{1}$ & $\langle[0.0,0.8],[0.1,0.9],[0.2,1.0],[-1.0,-0.0],[-1.0,-0.0],[-0.8,-0.0]\rangle$ \\
$P e_{2}$ & $\langle[1.0,1.0],[0.9,1.0],[1.0,1.0],[-0.9,-0.8],[-1.0,-0.9],[-0.9,-0.8]\rangle$ \\
$P e_{3}$ & $\langle[0.4,0.5],[0.2,0.4],[0.7,0.9],[-0.4,-0.2],[-0.9,-0.7],[-0.5,-0.4]\rangle$ \\
$P e_{4}$ & $\langle[0.8,0.9],[0.7,0.9],[0.8,0.9],[-0.9,-0.0],[-0.8,-0.7],[-0.8,-0.7]\rangle$ \\
\hline
\end{tabular}

Table 9. Interval valued bipolar neutrosophic decision matrix

Step 2. Decide weights of attributes.

The decision maker determined equal weights of attributes as follows:

$S_{1}=0.25, S_{2}=0.25, S_{3}=0.25$ and $S_{4}=0.25$.

Step 3. Determination of ideal sequence of the attributes values according to problem domain.

Here, decision maker constructed two types of the ideal sequence of attributes values; one for normal person and the other for person with bipolar respectively as follows:

$$
\begin{aligned}
& \operatorname{Id}^{*}{ }_{N}= \\
& \langle[0.5,0.5],[0.5,0.5],[0.5,0.5],[-0.5,-0.5],[-0.5,-0.5],[-0.5,-0.5]\rangle, \\
& \langle[0.5,0.5],[0.5,0.5],[0.5,0.5],[-0.5,-0.5],[-0.5,-0.5],[-0.5,-0.5]\rangle, \\
& \langle[0.5,0.5],[0.5,0.5],[0.5,0.5],[-0.5,-0.5],[-0.5,-0.5],[-0.5,-0.5]\rangle, \\
& \langle[0.5,0.5],[0.5,0.5],[0.5,0.5],[-0.5,-0.5],[-0.5,-0.5],[-0.5,-0.5]\rangle .
\end{aligned}
$$

$\operatorname{Id}^{*}{ }_{B}$

$\langle[0.0,1.0],[0.0,1.0],[0.0,1.0],[-1.0,-0.0],[-1.0,-0.0],[-1.0,-0.0]\rangle$,

$=\langle[0.0,1.0],[0.0,1.0],[0.0,1.0],[-1.0,-0.0],[-1.0,-0.0],[-1.0,-0.0]\rangle$,

$\langle\langle[0.0,1.0],[0.0,1.0],[0.0,1.0],[-1.0,-0.0],[-1.0,-0.0],[-1.0,-0.0]\rangle$,

$\langle[0.0,1.0],[0.0,1.0],[0.0,1.0],[-1.0,-0.0],[-1.0,-0.0],[-1.0,-0.0]\rangle$.

The previous sequence of attributes values for normal and bipolar state determined according to decision maker opinion and the problem domain, since in normal state the mood and behaviors of persons are balanced (i.e. not up nor down). So, we selected this sequence $[0.5,0.5],[0.5,0.5],[0.5,0.5],[-0.5,-0.5],[-0.5,-0.5],[-0.5,-0.5]$ under interval valued bipolar neutrosophic information. Also, in bipolar disease state, mood and behaviors of persons are unusually shifts from "down" and "up", then decision maker represented sequence of attribute values $[0.0,1.0],[0.0,1.0],[0.0,1.0],[-1.0,-0.0],[-1.0,-0.0],[-1.0,-0.0]$ under interval valued bipolar neutrosophic information.

Step 4. Calculate similarity measures.

Use Eqs. (22-24) for calculating similarity measures between alternatives (persons) and normal sequence of attributes values $I d^{*}{ }_{N}$, and also between alternatives and bipolar sequence of attributes values $I d^{*}{ }_{B}$ for identifying normal persons and others with bipolar disease as in Table 10. 


\begin{tabular}{|c|c|}
\hline $\begin{array}{c}\text { Similarity } \\
\text { measures }\end{array}$ & $\begin{array}{c}\text { Measure } \\
\text { values }\end{array}$ \\
\hline$C s_{1}{ }^{w}\left(P e_{1}, I d^{*}{ }_{N}\right)$ & 0.8325 \\
\hline$C s_{1}{ }^{w}\left(P e_{1}, I d^{*}{ }_{B}\right)$ & 0.9651 \\
\hline$C s_{1}{ }^{w}\left(P e_{2}, I d^{*}{ }_{N}\right)$ & 0.7771 \\
\hline$C s_{1}{ }^{w}\left(P e_{2}, I d^{*}{ }_{B}\right)$ & 0.7431 \\
\hline$C s_{1}{ }^{w}\left(P e_{3}, I d^{*}{ }_{N}\right)$ & 0.9649 \\
\hline$C s_{1}{ }^{w}\left(P e_{3}, I d^{*}{ }_{B}\right)$ & 0.8010 \\
\hline$C s_{1}{ }^{w}\left(P e_{4}, I d^{*}{ }_{N}\right)$ & 0.8445 \\
\hline$C s_{1}{ }^{w}\left(P e_{4}, I d^{*}{ }_{B}\right)$ & 0.8314 \\
\hline$C s_{2}{ }^{w}\left(P e_{1}, I d^{*}{ }_{N}\right)$ & 0.9238 \\
\hline$C s_{2}{ }^{w}\left(P e_{1}, I d^{*}{ }_{B}\right)$ & 0.9297 \\
\hline$C s_{2}{ }^{w}\left(P e_{2}, I d^{*}{ }_{N}\right)$ & 0.9238 \\
\hline$C s_{2}{ }^{w}\left(P e_{2}, I d^{*}{ }_{B}\right)$ & 0.7071 \\
\hline$C s_{2}{ }^{w}\left(P e_{3}, I d^{*}{ }_{N}\right)$ & 0.9617 \\
\hline$C s_{2}{ }^{w}\left(P e_{3}, I d^{*}{ }_{B}\right)$ & 0.8704 \\
\hline$C s_{2}{ }^{w}\left(P e_{4}, I d^{*}{ }_{N}\right)$ & 0.9238 \\
\hline$C s_{2}{ }^{w}\left(P e_{4}, I d^{*}{ }_{B}\right)$ & 0.7847 \\
\hline$C s_{3}{ }^{w}\left(P e_{1}, I d^{*}{ }_{N}\right)$ & 0.8500 \\
\hline$C s_{3}{ }^{w}\left(P e_{1}, I d^{*}{ }_{B}\right)$ & 0.9297 \\
\hline$C s_{3}{ }^{w}\left(P e_{2}, I d^{*}{ }_{N}\right)$ & 0.8090 \\
\hline$C s_{3}{ }^{w}\left(P e_{2}, I d^{*}{ }_{B}\right)$ & 0.7071 \\
\hline$C s_{3}{ }^{w}\left(P e_{3}, I d^{*}{ }_{N}\right)$ & 0.9563 \\
\hline$C s_{3}{ }^{w}\left(P e_{3}, I d^{*}{ }_{B}\right)$ & 0.8077 \\
\hline$C s_{3}{ }^{w}\left(P e_{4}, I d^{*}{ }_{N}\right)$ & 0.8526 \\
\hline$C s_{3}{ }^{w}\left(P e_{4}, I d^{*}{ }_{B}\right)$ & 0.7604 \\
\hline
\end{tabular}

Table 10. Similarity measures values and ranking order of alternatives

As appears in Table 10, the first person $P e_{1}$ suffers from bipolar disease and the rest are healthy. The three types of proposed cosine similarity measures have the same diagnosis results about $P e_{1}$ and its display the efficacy of this diagnosis.

\section{Conclusion and future directions}

In this research, we illustrated some drawbacks of existing measures of similarity for bipolar neutrosophic set. Since in bipolar and interval valued bipolar neutrosophic environments cosine similarity measure is yet to appear in literature, we proposed three types of cosine similarity measures under bipolar and interval valued bipolar neutrosophic sets. The basic properties of three proposed types are proved. Also, we proposed two methods for solving MADM problems based on proposed types of cosine similarity measures under bipolar and interval valued bipolar neutrosophic information. We also solved two numerical examples and compared results with other existing methods. Also, we applied proposed types of similarity measures in medical diagnosis of bipolar disorder disease.

Finally, we concluded that the proposed types of cosine similarity measures are better than other presented measures and cannot produce unreasonable (undefined) results, as we showed with some other existing methods. Also, the proposed measures are very simple, with the minimum computational burden as compared with other existing methods. 
In the near future, we will apply the suggested similarity measures in case-based reasoning systems to detect fraud.

\section{Conflict of interest}

Authors declare that there is no conflict of interest about the research.

\section{Funding}

This research has no funding source.

\section{Ethical approval}

This article does not contain any studies with human participants or animals performed by any of the authors.

\section{References}

[1] Zadeh, L. A. (1965). Fuzzy sets. Information and control, 8(3), 338-353.

[2] Atanassov, K. T. (1999). Intuitionistic fuzzy sets. In Intuitionistic fuzzy sets (pp. 1-137). Physica, Heidelberg.

[3] Florentin Smarandache, Neutrosophy. Neutrosophic Probability, Set, and Logic, ProQuest Information \& Learning, Ann Arbor, Michigan, USA, 105 p., 1998;http://fs.unm.edu/eBook-neutrosophics6.pdf

[4] Cheng, H. D., \& Guo, Y. (2008). A new neutrosophic approach to image thresholding. New Mathematics and Natural Computation, 4(03), 291-308.

[5] Guo, Y., \& Cheng, H. D. (2009). New neutrosophic approach to image segmentation. Pattern Recognition, 42(5), 587-595.

[6] Kharal, A. (2014). A neutrosophic multi-criteria decision making method. New Mathematics and Natural Computation, 10(02), 143-162.

[7] Liu, P., \& Wang, Y. (2014). Multiple attribute decision-making method based on single-valued neutrosophic normalized weighted Bonferroni mean. Neural Computing and Applications, 25(7-8), 2001-2010.

[8] Liu, P., \& Shi, L. (2015). The generalized hybrid weighted average operator based on interval neutrosophic hesitant set and its application to multiple attribute decision making. Neural Computing and Applications, 26(2), 457- 471.

[9] Majumdar, P., \& Samanta, S. K. (2014). On similarity and entropy of neutrosophic sets. Journal of Intelligent \& Fuzzy Systems, 26(3), 1245-1252.

[10] Peng, J. J., Wang, J. Q., Wang, J., Zhang, H. Y., \& Chen, X. H. (2016). Simplified neutrosophic sets and their applications in multi-criteria group decision-making problems. International journal of systems science, 47(10), 2342-2358. 
[11] Şahin, R., \& Küçük, A. (2015). Subsethood measure for single valued neutrosophic sets. Journal of Intelligent \& Fuzzy Systems, 29(2), 525-530.

[12] Haibin, Wang, F. Smarandache Y.Q. Zhang, et al., Single valuedneutrosophic sets,Multispace and Multistructure4(2010),410-413

[13] H. Wang, F. Smarandache Y.Q. Zhang et al.,Interval neutro-sophic sets and logic: Theory and applications in computing,Hexis, Phoenix, AZ 2005.

[14] Xu, Z. (2013). Intuitionistic fuzzy aggregation and clustering (Vol. 279). Springer.

[15] Ye, J. (2013). Multicriteria decision-making method using the correlation coefficient under single-valued neutrosophic environment. International Journal of General Systems, 42(4), 386-394.

[16] Ye, J. (2014). Similarity measures between interval neutrosophic sets and their applications in multicriteria decision-making. Journal of Intelligent \& Fuzzy Systems, 26(1), 165-172.

[17] Ye, J. (2014). Single valued neutrosophic cross-entropy for multicriteria decision making problems. Applied Mathematical Modelling, 38(3), 1170-1175.

[18] Ye, J. (2015). Trapezoidal neutrosophic set and its application to multiple attribute decision-making. Neural Computing and Applications, 26(5), 1157-1166.

[19] Ye, J. (2014). Some aggregation operators of interval neutrosophic linguistic numbers for multiple attribute decision making. Journal of Intelligent \& Fuzzy Systems, 27(5), 22312241.

[20] Zhang, H. Y., Wang, J. Q., \& Chen, X. H. (2014). Interval neutrosophic sets and their application in multicriteria decision making problems. The Scientific World Journal, 2014.

[21] Zhang, M., Zhang, L., \& Cheng, H. D. (2010). A neutrosophic approach to image segmentation based on watershed method. Signal Processing, 90(5), 1510-1517.

[22] Abdel-Basset, M., Mohamed, M., Smarandache, F., \& Chang, V. (2018). Neutrosophic Association Rule Mining Algorithm for Big Data Analysis. Symmetry, 10(4), 106.

[23] Abdel-Basset, M., Mohamed, M., Zhou, Y., \& Hezam, I. (2017). Multi-criteria group decision making based on neutrosophic analytic hierarchy process. Journal of Intelligent \& Fuzzy Systems, 33(6), 4055-4066.

[24] Abdel-Basset, M., Gunasekaran, M., Mohamed, M., \& Smarandache, F. (2018). A novel method for solving the fully neutrosophic linear programming problems. Neural Computing and Applications, 1-11.

[25] Abdel-Basset, M., Mohamed, M., \& Sangaiah, A. K. (2018). Neutrosophic AHPDelphi Group decision making model based on trapezoidal neutrosophic numbers. Journal of Ambient Intelligence and Humanized Computing, 9(5), 1427-1443. 
[26] Abdel-Basset, M., Mohamed, M., Hussien, A. N., \& Sangaiah, A. K. (2018). A novel group decision-making model based on triangular neutrosophic numbers. Soft Computing, 22(20), 6629-6643.

[27] Abdel-Basset, M., Zhou, Y., Mohamed, M., \& Chang, V. (2018). A group decision making framework based on neutrosophic VIKOR approach for e-government website evaluation. Journal of Intelligent \& Fuzzy Systems, 34(6), 4213-4224.

[28] Abdel-Basset, M., Gunasekaran, M., Mohamed, M., \& Chilamkurti, N. (2018). Threeway decisions based on neutrosophic sets and AHP-QFD framework for supplier selection problem. Future Generation Computer Systems.

[29] Mohamed, M.; Abdel-Baset, M.; Smarandache, F.; Zhou, Y. A Critical Path Problem in Neutrosophic Environment; Infinite Study: El Segundo, CA, USA, 2017

[30] Abdel-Basset, M., Mohamed, M., \& Smarandache, F. (2018). A hybrid neutrosophic group ANP-TOPSIS framework for supplier selection problems. Symmetry, 10(6), 226.

[31] Bosc, P., \& Pivert, O. (2013). On a fuzzy bipolar relational algebra. Information Sciences, 219, 1-16.

[32] Lee, K. M. (2000). Bipolar-valued fuzzy sets and their operations. In Proc. Int. Conf. on Intelligent Technologies, Bangkok, Thailand, 2000 (pp. 307-312).

[33] Lee, K. J. (2009). Bipolar fuzzy subalgebras and bipolar fuzzy ideals of BCK/BCIalgebras. Bull. Malays. Math. Sci. Soc, 32(3), 361-373.

[34] Chen, J., Li, S., Ma, S., \& Wang, X. (2014). -Polar Fuzzy Sets: An Extension of Bipolar Fuzzy Sets. The Scientific World Journal, 2014.

[35] Manemaran, S. V., \& Chellappa, B. (2010). Structures on bipolar fuzzy groups and bipolar fuzzy D-ideals under (T, S) norms. International Journal of Computer Applications, 9(12), 7-10.

[36] Majumder, S. K. (2012). Bipolar valued fuzzy sets in $\Gamma$-semigroups. Mathematica Aeterna, 2(3), 203-213.

[37] Zhou, M., \& Li, S. (2014). Application of bipolar fuzzy sets in semirings. Journal of Mathematical Research with Applications, 34(1), 61-72.

[38]Tamani, N. (2015, August). A bipolar approach for intuitionistic fuzzy alternative ranking. In 2015 IEEE International Conference on Fuzzy Systems (FUZZ-IEEE) (pp. 18). IEEE.

[39] Ezhilmaran, D., \& Sankar, K. (2015). Morphism of bipolar intuitionistic fuzzy graphs. Journal of Discrete Mathematical Sciences and Cryptography, 18(5), 605-621.

[40] Sankar, K., \& Ezhilmaran, D. (2016). Balanced bipolar intuitionistic fuzzy graphs. International Research Journal of Engineering and Technology (IRJET), 3(11), 806-812. 
[41] Deli, I., Ali, M., \& Smarandache, F. (2015, August). Bipolar neutrosophic sets and their application based on multi-criteria decision making problems. In 2015 International Conference on Advanced Mechatronic Systems (ICAMechS) (pp. 249-254). IEEE.

[42] Şahin, M., Deli, I., \& Uluçay, V. (2016). Jaccard vector similarity measure of bipolar neutrosophic set based on multi-criteria decision making. In: International conference on natural science and engineering (ICNASE'16), March 19-20, Kilis.

[43] Uluçay, V., Deli, I., \& Şahin, M. (2018). Similarity measures of bipolar neutrosophic sets and their application to multiple criteria decision making. Neural Computing and Applications, 29(3), 739-748.

[44] Deli, I., Yusuf, S., Smarandache, F., \& Ali, M. (2016). Interval valued bipolar neutrosophic sets and their application in pattern recognition. In IEEE World Congress on Computational Intelligence.

[45] Ye, J. (2015). Improved cosine similarity measures of simplified neutrosophic sets for medical diagnoses. Artificial intelligence in medicine, 63(3), 171-179.

[46] Deli, I., \& Subas, Y. A. (2016, May). Multiple criteria decision making method on single valued bipolar neutrosophic set based on correlation coefficient similarity measure. In International Conference on mathematics and mathematics education (ICMME-2016), Frat University, Elazg, Turkey (pp. 12-14).

[47] Pramanik, S., Dey, P., Smarandache, F., \& Ye, J. (2018). Cross entropy measures of bipolar and interval bipolar neutrosophic sets and their application for multi-attribute decision-making. Axioms, 7(2), 21.

[48] Mahmood, T.; Ye, J.; Khan, Q. Bipolar Interval Neutrosophic Set and Its Application in Multicriteria Decision Making. Available online: https://archive.org/details/BipolarIntervalNeutrosophicSet (accessed on 9 May 2019).

[49] Pramanik, S.; Dey, P.; Smarandache, F. Correlation Coefficient Measures of Interval Bipolar Neutrosophic Sets for Solving Multi-Attribute Decision Making Problems. Neutrosophic Sets Syst. 2018, 19, 70-79.

[50] Ma, H., Zhu, H., Hu, Z., Li, K., \& Tang, W. (2017). Time-aware trustworthiness ranking prediction for cloud services using interval neutrosophic set and ELECTRE. Knowledge-Based Systems, 138, 27-45.

[51] Liang, R., Wang, J., \& Zhang, H. (2017). Evaluation of e-commerce websites: An integrated approach under a single-valued trapezoidal neutrosophic environment. Knowledge-Based Systems, 135, 44-59.

[52] S. Broumi, A. Bakali, M. Talea, F. Smarandache and M. Ali, "Shortest Path Problem under Bipolar Neutrosphic Setting," Applied Mechanics and Materials, Vol. 859, 2016, pp. 59-66. 
[53] P. Arulpandy, M. Trinita Pricilla: Some Similarity and Entropy Measurements of Bipolar Neutrosophic Soft Sets, Neutrosophic Sets and Systems, vol. 25, 2019, pp. 174194. DOI: $10.5281 /$ zenodo. 2631523

[54] Mumtaz Ali, Le Hoang Son, Irfan Deli and Nguyen Dang Tien, Bipolar neutrosophic soft sets and applications in decision making, Journal of Intelligent \& Fuzzy Systems 33 (2017) 4077-4087

[55] Broumi, Said and Talea, Mohamed and Bakali, Assia and F. Smarandache and Ullah, Kifayat, Bipolar Neutrosophic Minimum Spanning Tree, Smart Application and Data Analysis for SmartCities (SADASC'18), 2018, pp.201-206. http://dx.doi.org/10.2139/ssrn.3127519

[56] Mullai, M., Broumi, S., Stephen, A.: Shortest path problem by minimal spanning tree algorithm using bipolar neutrosophic numbers. Int. J. Math. Trends Technol. 46(2), 80-87 (2017)

[57] Said Broumi, AssiaBakali, Mohamed Talea, FlorentinSmarandache, and RajkumarVerma, Computing Minimum Spanning Tree in Interval Valued Bipolar Neutrosophic Environment, International Journal of Modeling and Optimization, Vol. 7, No. $\quad 5, \quad 2017, p p 300-304, \quad$ DOI: $\quad$ 10.7763/IJMO.2017.V7.602, https://doi.org/10.1007/s41066-018-0084-7 